\title{
Dor, lesões e síndromes músculo- esqueléticas em adolescentes obesos versus eutróficos e sua relação com o uso de computadores e videogames
}

\author{
Suely Nóbrega Jannini
}

Dissertação apresentada ao Programa de Pós-Graduação da Faculdade de Medicina da Universidade de São Paulo para obtenção do título de Mestre em Ciências

Programa de Pediatria

Orientador: Prof. Dr. Clóvis Artur Almeida da Silva

São Paulo 
Dados Internacionais de Catalogação na Publicação (CIP)

Preparada pela Biblioteca da

Faculdade de Medicina da Universidade de São Paulo

Creprodução autorizada pelo autor

Jannini, Suely Nóbrega

Dor, lesões e síndromes músculo-esqueléticas em adolescentes obesos versus eutróficos e sua relação com o uso de computadores e vídeo-games / Suely Nóbrega Jannini. -- São Paulo, 2011.

Dissertação(mestrado)--Faculdade de Medicina da Universidade de São Paulo. Programa de Pediatria.

Orientador: Clóvis Artur Almeida da Silva.

Descritores: 1.Dor 2.Doenças músculosqueléticas 3.Obesidade 4.Adolescente 5.Computadores 6.Jogos de vídeo

USP/FM/DBD-130/11 
"OS FILHOS SÃO AS ÂNCORAS QUE PRENDEM A MÃE À VIDA."

Sófocles 


\section{Dedicatórias}

Para meu médico que ao jamais desistir de um paciente, permitiu através de seu conhecimento extenso e atualizado associado a sua ética inabalável, que eu reiniciasse minha vida acadêmica após 16 anos de esforços conjuntos.

A todos os médicos ilustres desconhecidos que assim procedem no seu diaa-dia, lutando a favor de seus pacientes contra doenças que se recusam a responder aos melhores medicamentos. 


\section{Agradecimentos}

Ao Prof. Dr. Clovis Artur Almeida da Silva, pela orientação durante todo o Mestrado com segurança, indiscutível competência científica e espírito humano, com muita dedicação e compreensão além de uma total disponibilidade mental para mim e para cada um dos seus orientandos. Difícil exprimir em palavras o reconhecimento que tenho pelo seu trabalho nos últimos 3 anos.

Ao Dr. Ulysses Dória Filho, pela inestimável contribuição quanto às análises estatísticas, ensinando desde como montar e preencher uma tabela Excell até como "traduzi-la" em linguagem escrita para a Dissertação. Não foi uma tarefa leve considerando o "cérebro feminino" e suas nuances estatísticas.

À Prof ${ }^{a}$ Dra. Magda Carneiro-Sampaio, pela confiança inabalável no término dessa empreitada, reforçando cada vez mais minha admiração que vem de quase 27 anos em seu valor como pessoa humana aliada à sua extensa produção científica.

Aos médicos da Unidade de Reumatologia do Instituto da Criança do Hospital das Clínicas, pelo incentivo. 


\begin{abstract}
À Mariza Kazue Umetsu Yoshikawa, da Biblioteca do Instituto da Criança, pelo sempre gentil e eficiente atendimento em todas solicitações de artigos.
\end{abstract}

Ao Nivaldo Lira Rocha, pelo incentivo, pelas palavras de acolhimento em todas as idas ao Instituto da Criança e por sua ajuda na parte gráfica.

Aos meus amigos Dr. Paolo Farris e Eliane Bernadino, pelas frequentes alterações nas escalas de sobreaviso do Hospital Infantil Jeser de Amarante Farias (HIJAF) para que assim pudesse cumprir os créditos do Mestrado além dos horários de orientação com o Professor Clóvis.

Ao Dr. Armando Lorga, Diretor Técnico dos Hospitais Regional Hans Dieter Schmidt e posteriormente do HIJAF, por não ter criado quaisquer dificuldades às minhas reposições das ausências nos horários de trabalho em entidades que ainda não têm nenhum incentivo para Mestrado ou Doutorado, desde que a carga horária e os atendimentos fossem respeitados.

À minha mãe (in memorian), que jamais deixou de acreditar em minha capacidade de estudo e esforço pessoal.

Ao meu pai (in memorian), que ao aprender a ler sem ajuda e ao se utilizar da matemática nas Gerências de lojas me provou que "o nordestino é antes de tudo um forte" (Euclides da Cunha). 
Às minhas irmãs Miriam e Márcia Nóbrega, que mesmo antes do falecimento da nossa mãe já haviam me adotado como filha, cuidando como um bem muito frágil, algo que persiste até hoje apesar do distanciamento geográfico entre nós três.

À minha querida prima Lúcia Medeiros Ueno, que tantas vezes me acolheu em sua casa durante as idas a São Paulo, deixando seu filho caçula (Fábio) no quarto de hóspedes.

Aos meus gatos de estimação, pela companhia na saúde e na doença, de dia e de noite, sem exceção: Hamon Felipe (que nunca deixou que eu perdesse a hora), Eduarda Gabriela "Duda" (companheira de todos os momentos, apesar de suas supostas enxaquecas, causando mau humor quase constante), Gabrielly Patrícia "Bibi" (perdida por uma falha profissional veterinária, cujas sonecas junto comigo são inesquecíveis) e Frederica "Kika", que nos adotou e nos diverte até hoje com suas aventuras.

Às minhas filhas, que apesar de virem por último, com certeza estão no topo da minha lista de prioridades em agradecimentos e afeto. Marcela, incansável em seu apoio constante como braço direito em tudo; Flávia, também incansável em observar a mãe e detectar sinais de tristeza, cansaço ou exaustão, me cobrindo de atenções e carinhos e Isabela, apesar de 
odiando a palavra Mestrado desde intra-útero, ajudando em tudo que foi solicitado sempre carinhosamente. Aprendi quase tudo de informática com vocês. Não desistam: "velhinhos também aprendem". Vocês são o melhor time para se viver. Não se separem nunca.

Ao meu esposo José Francisco Jannini, que também não entendendo a razão do Mestrado, aceitou meu desejo de "eterna estudante" e facilitou ao máximo esta missão assumindo uma série de funções que eram minhas e apoiando financeiramente melhor que o CNPq. Tranquilo, nem o mestrado é ou será concorrente para você.

Se vocês quatro não existissem, nada faria sentido. 


\title{
ÍNDICE
}

\author{
RESUMO
}

ABSTRACT

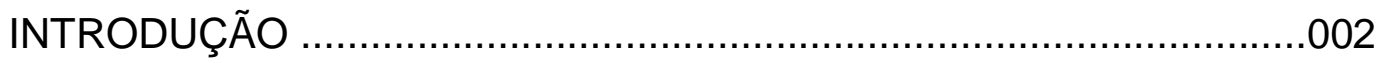

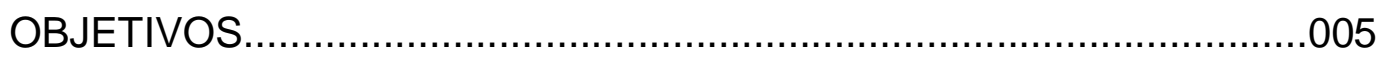

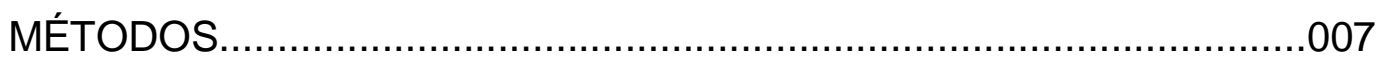

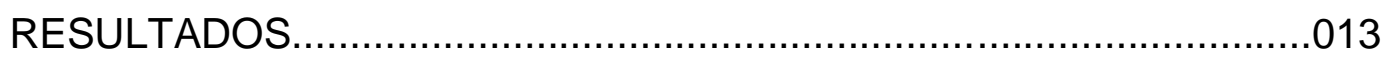

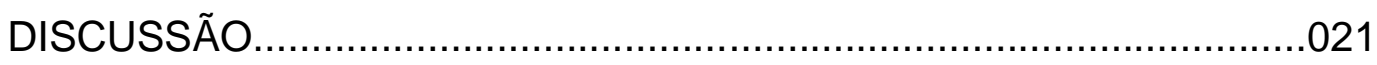

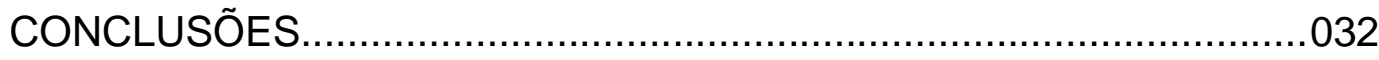

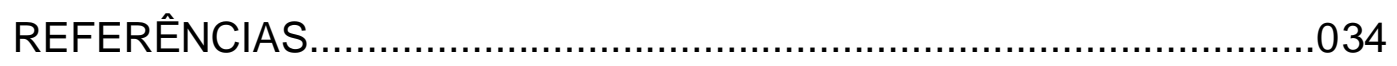




\section{Resumo}

Objetivo: Avaliar presença de dor, síndromes músculo-esqueléticas, alterações ortopédicas e uso de computador e videogame em adolescentes.

Métodos: Um estudo transversal avaliou 100 adolescentes consecutivos com obesidade e 100 eutróficos a partir de um questionário confidencial, auto-aplicável, incluindo dados demográficos, prática esportiva, sintomas dolorosos do sistema músculo-esquelético e uso de computador e videogame. Pré-teste e re-teste do questionário foram realizados. O exame físico avaliou seis síndromes músculo-esqueléticas e sete alterações ortopédicas.

Resultados: O índice de Kappa entre pré-teste e re-teste foi de 0,724. Dor e síndromes músculo-esqueléticas foram igualmente prevalentes nos dois grupos ( $44 \%$ vs. 56\%, $p=0,09 ; 12 \%$ vs. $16 \%, p=0,541$; respectivamente). Entretanto, alterações ortopédicas ( $98 \%$ vs. $76 \%, p=0,0001)$, encurtamento de quadríceps ( $89 \%$ vs. $44 \%, p=0,0001)$ e geno valgo ( $87 \%$ vs. $24 \%$, $\mathrm{p}=0,0001$ ) foram significativamente mais evidenciados nos obesos versus controles. As medianas do tempo de uso do computador no dia anterior a pesquisa, nos sábados e domingos foram menores nos obesos (30 vs. 60 minutos, $p=0,0001$; 1 vs. 60 minutos, $p=0,001$; 0 vs. 30 minutos, $p=0,02$; respectivamente). Uso de minigame foi menor nos obesos ( $2 \%$ vs. $11 \%$, $\mathrm{p}=0,003$ ), não havendo diferença no uso de videogame nos dois grupos ( $p>0,05)$. Comparações entre obesos com e sem dor evidenciaram maior frequência no gênero feminino ( $59 \%$ vs. $39 \%, p=0,048)$ e maior mediana de tempo de uso nos domingos [0 (0-720) vs. 0 (0-240)minutos, $p=0.028]$.

Conclusões: Obesidade pode causar danos ao sistema ósteo-articular no início da adolescência, principalmente nos membros inferiores. Programas específicos para adolescentes obesos do sexo feminino com dor músculoesquelética precisam ser desenvolvidos.

Palavras-chave: adolescente, obesidade, dor, síndrome musculoesqulética, computador, videogame. 


\section{Abstract}

Objective: To evaluate the presence of pain, musculoskeletal syndromes and orthopedic abnormalities and the use of computers and videogames in adolescents.

Methods: A cross-sectional study using a self-applicable questionnaire to assess 100 obese adolescents and 100 healthy controls included demographic data, sports, musculoskeletal painful symptoms and the use of computer and videogames. A pretest and its replication were performed. The physical examination evaluated six musculoskeletal syndromes and seven orthopedics alterations.

Results: The Kappa index between the pretest and its replication was 0.724. Pain and musculoskeletal syndromes were similar in both groups (44\% vs. $56 \%, p=0.09 ; 12 \%$ vs. $16 \%, p=0.541$; respectively). However, orthopedics alterations (98\% vs. $76 \%, p=0.0001)$, tight quadriceps $(89 \%$ vs. $44 \%$, $p=0.0001)$ and genu valgum ( $87 \%$ vs. $24 \%, p=0.0001)$ were significantly observed in obese versus controls. The median time spent using computer on the prior to the research and on Saturdays and Sundays were lower in obese (30 vs. 60minutes, $p=0.0001 ; 1$ vs. 60minutes, $p=0.001 ; 0$ vs. 30 minutes, $p=0.02$; respectively). Minigame use was lower in obese ( $2 \%$ vs. $11 \%, p=0.003$ ), without any differences in the use of videogames in both groups ( $p>0.05$ ). Comparisons between obese adolescents with and without musculoskeletal pain demonstrated higher frequency in females (59\% vs. $39 \%, p=0.048)$.

Conclusions: Obesity can induce damages to the osteoarticular system at the beginning of the adolescence, mainly in the inferior limbs. Specific programs for female obese adolescents with musculoskeletal pain should be established.

Key-words: adolescent, obesity, pain, musculoskeletal syndrome, computer, video game. 


\section{Introdução}

Obesidade e sobrepeso na adolescência são indiscutíveis problemas mundiais de saúde pública ${ }^{1-4}$, inclusive no Brasil. Wang et al., $2002^{5}$ avaliaram a prevalência de sobrepeso em quatro países (Brasil, Rússia, China e Estados Unidos da América) nas últimas décadas e constataram um incremento importante no Brasil de $240 \%$.

A obesidade na adolescência é uma doença crônica multifatorial e está relacionada a fatores genéticos ${ }^{4}$, mudanças dos padrões alimentares, redução da atividade física e incremento da utilização de computador e videogames, entre outros fatores ${ }^{3}$. Adolescentes obesos têm maior prevalência de co-morbidades e complicações, tais como: hipertensão arterial, dislipidemia, intolerância à glicose ${ }^{1,2,4}$, câncer, doenças pulmonares e dermatológicas, assim como: alterações gastrintestinais, gênito-urinárias e neuropsiquiátricas, entre outras ${ }^{1,2}$.

Alguns estudos evidenciaram que adolescentes obesos apresentam também uma maior prevalência de dor músculo-esquelética localizada ${ }^{6-11}$, principalmente em membros inferiores ${ }^{6,10,11}$ e em região lombar ${ }^{9,10-12}$ assim como alterações ortopédicas localizadas ${ }^{12,13:}$ geno valgo, geno varo ${ }^{11,13}$, geno recurvatum e encurtamento de quadríceps ${ }^{9}$. Síndromes músculoesqueléticas que apresentam dor crônica e difusa como fibromialgia juvenil, foram raramente estudadas nos adolescentes obesos ${ }^{9}$. 
Outros estudos também evidenciaram associação entre uso de computador e dor músculo-esquelética em adolescentes e jovens eutróficos $^{14}$, tais como: dor em membros superiores ${ }^{15}$, dor cervical ${ }^{15-17}$ e sensação de desconforto músculo-esquelético ${ }^{18,19}$. Em contraste, outros não evidenciaram associação entre diversos tipos de dor com o uso de computador em adolescentes eutróficos ${ }^{20,21}$ e para o nosso conhecimento não há estudos avaliando estes aspectos em adolescentes obesos.

Ainda não há pesquisa que tenha estudado simultaneamente dor, síndromes músculo esqueléticas, alterações ortopédicas e uso de computador e videogame em adolescentes obesos versus eutróficos.

Além disto, nenhum estudo avaliou sistematicamente a prevalência de síndrome de hipermobilidade articular benigna (SHAB), síndrome miofascial, tendinites, bursites e epicondilites em adolescentes com esta doença crônica (Obesidade). 


\section{Objetivos}

1. Determinar a presença de dor, síndromes músculoesqueléticas, alterações ortopédicas, uso de computador e videogame em adolescentes obesos e eutróficos.

2. Avaliar possível associação entre dados demográficos, síndromes, alterações ortopédicas e uso de computador e videogame entre adolescentes obesos com dor versus adolescentes obesos sem dor músculo-esquelética. 
MÉTODOS 


\section{Métodos}

\section{População do estudo}

Duzentos adolescentes consecutivos (100 adolescentes com obesidade e 100 eutróficos) do Ambulatório de Adolescentes do Hospital Regional Hans Dieter Schmidt (HRHDS), Joinville, Santa Catarina, foram avaliados durante o período de 2008 a 2010. O estudo foi aprovado pelas Comissões de Pesquisa e Ética do HRHDS e do Hospital das Clinicas da Faculdade de Medicina da Universidade de São Paulo (HC-FMUSP). Todos os adolescentes obesos e eutróficos, assim como os seus respectivos familiares, assinaram o termo de consentimento livre e esclarecido.

Obesidade foi definida de acordo com o índice de massa corpórea (IMC) maior ou igual ao percentil 95 National Control Health System (NCHS) de 2000 para sexo e idade. Eutrofia foi definido quando o IMC fosse menor ou igual ao percentil 85 e maior que o percentil 5 do NCHS para sexo e idade $^{22}$.

Os critérios de inclusão foram: adolescência (idades entre 10 e 19 anos e 11 meses) e aceitação da participação do estudo pelos adolescentes e pais (ou seus responsáveis legais). Os pacientes com dor e lesões músculo esqueléticos secundárias às seguintes doenças foram excluídos: do colágeno, infecciosas, onco-hematológicas, genéticas, endócrinas (como 
doenças tireoidianas e diabetes mellitus) e traumáticas recentes. Além disto, os pacientes que utilizavam medicamentos (tais como glicocorticoides, anticonvulsivantes e anti-psicóticos) e os que haviam preenchido com qualidade inadequada o questionário foram também excluídos.

\section{Metodologia}

A pesquisa consistiu em estudo transversal e incluiu aplicação de questionário e de exame físico geral e específico do sistema músculoesquelético por um profissional treinado (pesquisadora).

O questionário foi confidencial, auto-aplicável e incluiu os seguintes aspectos: dados demográficos, prática esportiva, sintomas dolorosos do sistema músculo-esquelético presentes nos últimos três meses e questões relacionadas ao uso de computador e videogame ${ }^{21,23,24}$ (Anexo 1). As classes socioeconômicas das famílias em que os adolescentes estavam inseridos foram avaliadas de acordo com a classificação da Associação Brasileira dos Institutos de Pesquisa de Mercados ${ }^{25}$.

Entre agosto e setembro de 2008, pré-teste e re-teste do questionário foram realizados em 30 adolescentes consecutivos (15 obesos e 15 eutróficos) com intervalo de 10 dias para avaliação da confiabilidade nas respostas. 
O questionário incluiu 70 questões e uma figura representativa do corpo para localizar a respectiva dor, conforme utilizado e publicado anteriormente ${ }^{21,23,24}$. Dor músculo-esquelética foi definida como aqueles que responderam "sim" à presença de dor em qualquer localização nos últimos três meses da pesquisa. Os seguintes tipos de dor músculo-esquelética foram também sistematicamente avaliados: dor cervical, dor em membros superiores, dor torácica, dor em músculo trapézio, dor lombar e dor localizada em membros inferiores ${ }^{23}$. Com relação à utilização do computador, o questionário avaliou os seguintes aspectos: uso do computador, disponibilidade domiciliar, existência de aparelho próprio, utilização de aparelho portátil (laptop), emprego de teclado, mouse ou outro. Além disto, incluiu avaliação de: idade de início do uso do computador em anos, tempo de uso deste em dias por semana, tempo de uso no dia anterior a pesquisa, tempo de uso nos sábados e nos domingos. $O$ mesmo foi relatado em relação à utilização de videogame e minigame/gameboy ${ }^{21}$.

O exame físico geral incluiu avaliação do índice de massa corpórea (IMC - peso em quilograma dividido por altura ao quadrado, $\mathrm{Kg} / \mathrm{m}^{2}$ ) e avaliação da circunferência abdominal (em centímetros, cm). As pressões arteriais sistólicas (PAS) e diastólicas (PAD) foram aferidas em milímetros de mercúrio, mmHg. Hipertensão arterial sistêmica foi definida como PAS e/ou PAD superior ou igual ao percentil 95 para o sexo, idade e estatura ${ }^{26}$.

Com relação ao exame músculo-esquelético, as seguintes síndromes músculo-esqueléticas foram avaliadas: fibromialgia juvenil, síndrome de 
hipermobilidade articular benigna (SHAB), síndrome miofascial, tendinites, bursites e epicondilites. Avaliação ortopédica da coluna e dos membros inferiores foi realizada para pesquisa de: escoliose, geno valgo, geno varo, geno recurvatum, encurtamento de quadríceps, encurtamento de isquiotibiais e hálux valgo ${ }^{27}$.

A fibromialgia juvenil foi diagnosticada segundo os critérios do Colégio Americano de Reumatologia pela demonstração de 11 dos 18 pontos dolorosos, associados à presença de dor difusa, com duração mínima de três meses ${ }^{28}$. Hipermobilidade articular $(\mathrm{HA})$ foi definida de acordo com os critérios de Beighton. A SHAB foi descrita como a presença de HA associada à dor no sistema músculo-esquelético e presença de 5 dos 9 critérios $^{29}$. A síndrome miofascial foi diagnosticada pela presença de ponto de gatilho ativo, definido como um ponto doloroso localizado em uma banda tensa muscular. Ao ser pressionado, este induziria uma dor referida em áreas padronizadas e reprodutíveis para cada músculo ${ }^{23}$. A atividade do ponto de gatilho foi definida pela confirmação do adolescente da existência anterior dessa dor. Os pontos foram pesquisados nos grupos musculares acometidos em lesões por esforços repetitivos e nos que participam ativamente dos movimentos necessários para uso de computador e videogame. Os seguintes músculos ou grupos musculares foram avaliados bilateralmente: trapézio, subescapular, musculatura cervical posterior, bíceps, tríceps, braquioradial, musculatura extensora e flexora da mão, punho e dedos e musculatura intrínseca da mão ${ }^{23}$. 
Além disto, com relação ao exame esquelético com o paciente em posição supina, encurtamento dos isquiotibiais foi avaliado com 0 posicionamento do quadril a $90^{\circ}$ e extensão do joelho, estando presente se fosse inferior a $15^{\circ}$. Com o paciente em pronação, a flexibilidade do quadríceps foi avaliada com o joelho completamente estendido, com a face anterior da coxa tocando a superfície de contato. O quadríceps era encurtado se os calcâneos não atingissem as nádegas ${ }^{27}$.

\section{Análise estatística}

Os resultados foram apresentados em mediana (variação) ou média \pm desvio-padrão para variáveis contínuas e número (\%) para variáveis categóricas. As medianas dos resultados foram comparadas pelo teste de Mann-Whitney para variáveis contínuas para determinar diferenças entre adolescentes obesos versus eutróficos e entre adolescentes obesos com dor versus adolescentes obesos sem dor. Para as variáveis categóricas, as diferenças foram calculadas pelo teste exato de Fisher e qui-quadrado. Valores de $p<0,05$ foram considerados estatisticamente significantes. Para avaliar a confiabilidade do questionário entre o pré-teste e o re-teste foi utilizado o índice de Kappa. 
RESULTADOS 


\section{Resultados}

O índice de Kappa entre o pré-teste e re-teste foi de 0,724 , demonstrando uma excelente confiabilidade nas respostas dos adolescentes obesos e eutróficos.

\section{Adolescentes obesos versus eutróficos}

As medianas do IMC e da circunferência abdominal foram significativamente maiores em adolescentes obesos versus eutróficos [29,45 $(23,7-45,4)$ vs. $18,8(14,8-24,1) \mathrm{kg} / \mathrm{m}^{2}, \mathrm{p}=0,0001 ; 95$ (69,5-123) vs. 69 (5583) $\mathrm{cm}, p=0,0001$; respectivamente]. Além disto, as medianas das pressões arterial sistólica e diastólica foram significativamente maiores em adolescentes obesos e eutróficos [110 (80-140) vs. 100 (70-130) mmHg, $\mathrm{p}=0,0001 ; 70$ (50-90) vs. $60(40-80) \mathrm{mmHg}, \mathrm{p}=0,0001$; respectivamente]. Não houve diferença estatística entre idade atual, gênero feminino, classes sócio-econômicas $\mathrm{C}$ e D e prática esportiva (Tabela 1).

As presenças de pelo menos uma dor músculo-esquelética e de pelo menos uma síndrome músculo-esquelética foram semelhantes em pacientes obesos versus eutróficos ( $44 \%$ vs. $56 \%, p=0,09 ; 12 \%$ vs. $16 \%, p=0,541$; respectivamente). Dor torácica e epicondilite foram significativamente 
menores em obesos versus controles ( $2 \%$ vs. $13 \%, p=0,005 ; 3 \%$ vs. $11 \%$, $p=0,027$; respectivamente). Pelo menos uma alteração ortopédica, encurtamento de quadríceps ou geno valgo foi significativamente mais evidenciada nos obesos comparados aos controles ( $98 \%$ vs. $76 \%, p=0,0001$; $89 \%$ vs. $44 \%, p=0,0001 ; 87 \%$ vs. $24 \%, p=0,0001$; respectivamente] (Tabela 1).

As características do uso de computador, videogame e minigame em adolescentes obesos versus eutróficos estão na Tabela 2. As frequências de disponibilidade domiciliar e do uso de computador no dia anterior a pesquisa foram significativamente menores nos obesos comparados aos eutróficos (54\% vs. $68 \%, p=0,042 ; 32 \%$ vs. $48 \%, p=0,015$; respectivamente). Além disto, as medianas da idade de início do uso, tempo de uso no dia anterior à pesquisa, tempo de uso no sábado e no domingo foram também significativamente menores nos obesos versus controles [10 (5-15) vs. 11 (516) anos, $p=0,01 ; 30(0-360)$ vs. 60 (0-720) minutos, $p=0,0001 ; 1(0-720)$ vs. $60(0-600)$ minutos, $p=0,001 ; 0(0-720)$ vs. 30 (0-720) minutos, $p=0,02$; respectivamente]. Não houve diferença em relação ao uso de videogame e minigame nos dois grupos (Tabela 2). 
Adolescentes obesos que referiam dor músculo-esquelética versus os sem dor

Com relação às comparações entre adolescentes obesos que referiam dor músculo-esquelética versus os que não referiam, o gênero feminino teve mais freqüentemente dor ( $59 \%$ vs. $39 \%, p=0,048)$. Não houve diferença estatística entre idade atual, classes sócio-econômicas C e D, IMC, circunferência abdominal, PA e prática esportiva nos dois grupos estudados (Tabela 3). Apesar de uma maior freqüência de síndromes músculo-esqueléticas nos adolescentes obesos com dor, não houve diferença entre esses e os que não referiam dor $(p>0,05)$. Quatro adolescentes obesos tinham tendinite/epicondilite, mas não referiram dor no questionário (Tabela 3). Outras alterações ortopédicas foram similares em adolescentes obesos com dor versus sem dor (Tabela 3).

Apenas a mediana do tempo de uso do vídeo-game no domingo foi significativamente maior em obesos que reportavam dor versus obesos que não referiam dor [0 (0-720) vs. 0 minutos $(0-240), p=0,028]$ (Tabela 4). Não houve diferença significativa entre as outras variáveis do videogame, assim como do computador e minigame em obesos com dor versus obesos sem dor (Tabela 4). 
Tabela 1 - Dados demográficos, dados antropométricos, pressão arterial (PA), prática esportiva, síndromes músculo-esqueléticas e alterações ortopédicas em adolescentes obesos versus eutróficos.

\begin{tabular}{|c|c|c|c|}
\hline Variáveis & $\begin{array}{l}\text { Obesos } \\
(n=100)\end{array}$ & $\begin{array}{l}\text { Eutróficos } \\
(n=100)\end{array}$ & $\mathbf{P}$ \\
\hline \multicolumn{4}{|l|}{ Dados demográficos } \\
\hline Idade atual, anos & $12,6(10-18,2)$ & $13(10-19)$ & 0,051 \\
\hline Gênero feminino & $48(52)$ & $60(60)$ & 0,089 \\
\hline Classe socioeconômica, C e D & $71(71)$ & $62(62)$ & 0,231 \\
\hline \multicolumn{4}{|l|}{ Dados antropométricos } \\
\hline $\mathrm{IMC}, \mathrm{kg} / \mathrm{m}^{2}$ & $29,45(23,7-45,4)$ & $18,8(14,8-24,1)$ & 0,0001 \\
\hline Circunferência abdominal, cm & $95(69,5-123)$ & $69(55-83)$ & 0,0001 \\
\hline \multicolumn{4}{|l|}{ PA } \\
\hline Hipertensão arterial sistêmica & $19(21)$ & $3(3)$ & 0,0004 \\
\hline PA sistólica, mmHg & $110(80-140)$ & $100(70-130)$ & 0,0001 \\
\hline PA diastólica, $\mathrm{mmHg}$ & $70(50-90)$ & $60(40-80)$ & 0,0001 \\
\hline \multicolumn{4}{|l|}{ Prática esportiva } \\
\hline Educação física $\geq 2$ vezes/sem & $92(92)$ & $92(92)$ & 1,0 \\
\hline Esportes $\geq 2$ vezes/semana & $26(26)$ & $27(27)$ & 1,0 \\
\hline Dor músculo-esquelética & $44(44)$ & $56(56)$ & 0,09 \\
\hline Dor cervical & $6(6)$ & $10(10)$ & 0,435 \\
\hline Dor em membros superiores & $3(3)$ & $10(10)$ & 0,082 \\
\hline Dor torácica & $2(2)$ & $13(13)$ & 0,005 \\
\hline Dor em músculo trapézio & $16(16)$ & $17(17)$ & 1,0 \\
\hline Dor lombar & $0(0)$ & $4(4)$ & 0,121 \\
\hline Dor em membros inferiores & $23(23)$ & $22(22)$ & 1,0 \\
\hline $\begin{array}{l}\text { Síndromes músculo- } \\
\text { esqueléticas }\end{array}$ & $12(12)$ & $16(16)$ & 0,541 \\
\hline Fibromialgia juvenil & $0(0)$ & $1(1)$ & 1,0 \\
\hline SHAB & $0(0)$ & $2(2)$ & 0,497 \\
\hline Síndrome miofascial & $0(0)$ & $1(1)$ & 1,0 \\
\hline Tendinite & $9(9)$ & $3(3)$ & 0,134 \\
\hline Epicondilite & $3(3)$ & $11(11)$ & 0,027 \\
\hline Bursite & $0(0)$ & $0(0)$ & 1,0 \\
\hline Alterações ortopédicas & $98(98)$ & $76(76)$ & 0,0001 \\
\hline Escoliose & $30(30)$ & 33 (33) & 0,761 \\
\hline Encurtamento de quadríceps & $89(89)$ & $44(44)$ & 0,0001 \\
\hline Geno valgo & $87(87)$ & $24(24)$ & 0,0001 \\
\hline Geno varo & $1(1)$ & $8(8)$ & 0,0349 \\
\hline Geno recurvatum & $1(1)$ & $0(0)$ & 1,0 \\
\hline Encurtamento de isquiotibiais & $6(6)$ & $1(1)$ & 0,054 \\
\hline Hálux valgo & $1(1)$ & $0(0)$ & 1,0 \\
\hline
\end{tabular}


Tabela 2 - Características do uso de computador, vídeo-game (VG) e minigame (MG) em adolescentes obesos versus eutróficos.

\begin{tabular}{lccc}
\hline Variáveis & $\begin{array}{c}\text { Obesos } \\
(\mathrm{n}=100)\end{array}$ & $\begin{array}{c}\text { Eutróficos } \\
(\mathrm{n}=100)\end{array}$ & $\mathbf{P}$ \\
\hline Computador & $69(69)$ & $79(79)$ & 0,107 \\
Uso do computador & $54(54)$ & $68(68)$ & $\mathbf{0 , 0 4 2}$ \\
Disponibilidade domiciliar & $24(24)$ & $36(36)$ & 0,064 \\
Uso de computador próprio & $8(8)$ & $14(14)$ & 0,233 \\
Uso de Laptop próprio & $32(32)$ & $48(48)$ & $\mathbf{0 , 0 1 5}$ \\
Uso no dia anterior & $10(5-15)$ & $11(5-16)$ & $\mathbf{0 , 0 1}$ \\
Idade de início do uso, anos & $0(0-360)$ & $60(0-720)$ & $\mathbf{0 , 0 0 0 1}$ \\
Tempo de uso no dia anterior, min & $1(0-720)$ & $60(0-600)$ & $\mathbf{0 , 0 0 1}$ \\
Tempo de uso nos sábados, min & $0(0-720)$ & $30(0-720)$ & $\mathbf{0 , 0 2}$ \\
Tempo de uso nos domingos, min & $2(0-7)$ & $3(0-7)$ & 0,077 \\
Tempo de uso na semana, dias & & & \\
VG & $39(39)$ & $43(43)$ & 0,605 \\
Uso do VG & $32(32)$ & $35(35)$ & 0,617 \\
Disponibilidade domiciliar & $21(21)$ & $24(24)$ & 0,638 \\
Uso de VG próprio & $12(12)$ & $12(12)$ & 1,0 \\
Uso do VG no dia anterior & $0(0-720)$ & $0(0-360)$ & 0,459 \\
Tempo de uso no dia anterior, min & $0(0-720)$ & $0(0-720)$ & 0,933 \\
Tempo de uso no sábado, min & $0(0-720)$ & $0(0-720)$ & 0,833 \\
Tempo de uso no domingo, min & $0(0-7)$ & $0(0-7)$ & 0,65 \\
Tempo de uso na semana, dias & & & \\
MG & $2(2)$ & $11(11)$ & $\mathbf{0 , 0 0 3}$ \\
Uso do MG & $2(2)$ & $3(3)$ & 1,0 \\
Uso do MG no dia anterior & $0(0-5)$ & $0(0-7)$ & 0,035 \\
Tempo de uso na semana, dias & $0(0-15)$ & $0(0-720)$ & 0,637 \\
Tempo de uso no dia anterior, min & $0(2)$
\end{tabular}

Os resultados são apresentados em $\mathrm{n}(\%)$ e mediana (variação), min - minutos. 
Tabela 3 - Dados demográficos, dados antropométricos, pressão arterial (PA), prática esportiva, síndromes músculo-esqueléticas e alterações ortopédicas em adolescentes obesos com dor versus obesos sem dor.

\begin{tabular}{|c|c|c|c|}
\hline Variáveis & $\begin{array}{c}\text { Obesos com } \\
\text { dor } \\
(n=44)\end{array}$ & $\begin{array}{c}\text { Obesos sem } \\
\text { dor } \\
(n=56)\end{array}$ & $\mathbf{P}$ \\
\hline \multicolumn{4}{|l|}{ Dados demográficos } \\
\hline Idade atual, meses & $12,5(10-19)$ & $12,7(10,2-17,2)$ & 0,692 \\
\hline Gênero feminino & $26(59)$ & $22(39)$ & 0,048 \\
\hline Classe socioeconômica, C ou D & 32 (73) & $39(70)$ & 0.829 \\
\hline \multicolumn{4}{|l|}{ Dados antropométricos } \\
\hline $\mathrm{IMC}, \mathrm{kg} / \mathrm{m}^{2}$ & $29,2(23,7-43,5)$ & $29,5(24,1-45,4)$ & 0,981 \\
\hline $\begin{array}{l}\text { Circunferência abdominal, cm } \\
\text { PA }\end{array}$ & $94(69,5-117)$ & $96,25(82-123)$ & 0,352 \\
\hline Hipertensão arterial sistêmica & \multicolumn{3}{|c|}{ PA } \\
\hline PA sistólica, $\mathrm{mmHg}$ & $110(80-140)$ & $110(80-130)$ & 0,432 \\
\hline PA diastólica, mmHg & $70(50-90)$ & $70(50-90)$ & 0,945 \\
\hline \multicolumn{4}{|l|}{ Prática esportiva } \\
\hline Educação física $\geq 2$ vezes/semana & $41(93)$ & $51(91)$ & 1,0 \\
\hline Esportes $\geq 2$ vezes/semana & $11(25)$ & $15(27)$ & 1,0 \\
\hline Síndromes músculo-esqueléticas & $8(18)$ & $4(7)$ & 0,124 \\
\hline Fibromialgia juvenil & $0(0)$ & $0(0)$ & 1,0 \\
\hline SHAB & $0(0)$ & $0(0)$ & 1,0 \\
\hline Síndrome miofascial & $0(0)$ & $0(0)$ & 1,0 \\
\hline Tendinite & $6(14)$ & $3(5)$ & 0,176 \\
\hline Epicondilite & $2(4)$ & $1(2)$ & 0,581 \\
\hline Alterações ortopédicas & $44(100)$ & $54(96)$ & 0,502 \\
\hline Escoliose & $11(25)$ & $19(34)$ & 0,384 \\
\hline Encurtamento de quadríceps & $39(89)$ & $50(89)$ & 1,0 \\
\hline Geno valgo & $41(93)$ & $46(82)$ & 0,1380 \\
\hline Geno varo & $1(2)$ & $0(0)$ & 0,44 \\
\hline Geno recurvatum & $1(2)$ & $0(0)$ & 0,44 \\
\hline Encurtamento de ísquiotibiais & $1(2)$ & $5(9)$ & 0,225 \\
\hline Hálux valgo & $1(2)$ & $0(0)$ & 0,44 \\
\hline
\end{tabular}

Os resultados são apresentados em n (\%) e mediana (variação), SHAB - síndrome de hipermobilidade articular benigna. 
Tabela 4 - Características do uso de computador, vídeo-game (VG) e mini-game (MG) em adolescentes obesos com dor versus obesos sem dor.

\begin{tabular}{|c|c|c|c|}
\hline Variáveis & $\begin{array}{l}\text { Obesos } \\
\text { com dor } \\
(n=44)\end{array}$ & $\begin{array}{c}\text { Obesos sem } \\
\text { dor } \\
(n=56)\end{array}$ & $\mathbf{P}$ \\
\hline \multicolumn{4}{|l|}{ Computador } \\
\hline Uso do computador & $30(68)$ & $39(70)$ & 1,0 \\
\hline Disponibilidade domiciliar & $24(54)$ & $30(54)$ & 1,0 \\
\hline Uso de computador próprio & $12(27)$ & $12(21)$ & 0,638 \\
\hline Uso de Laptop próprio & $6(14)$ & $2(4)$ & 0,133 \\
\hline Uso no dia anterior & $12(27)$ & $20(36)$ & 0,396 \\
\hline Idade de início do uso, anos & $10(5-10,8)$ & $10(3-15)$ & 0,734 \\
\hline Tempo de uso no dia anterior, min & $20(0-360)$ & $30(0-300)$ & 0,558 \\
\hline Tempo de uso nos sábados, min & $1(0-720)$ & $45(0-720)$ & 0,14 \\
\hline Tempo de uso nos domingos, min & $1(0-720)$ & $30(0-720)$ & 0,646 \\
\hline \multicolumn{4}{|l|}{ VG } \\
\hline Uso do VG & $17(39)$ & $22(39)$ & 1,0 \\
\hline Disponibilidade domiciliar & $16(36)$ & $16(29)$ & 0,518 \\
\hline Uso de VG próprio & $9(21)$ & $12(21)$ & 1,0 \\
\hline Uso do VG no dia anterior & $5(11)$ & $7(13)$ & 1,0 \\
\hline Tempo de uso no dia anterior, min & $0(0-720)$ & $0(0-120)$ & 0,123 \\
\hline Tempo de uso nos sábados, min & $0(0-720)$ & $0(0-180)$ & 0,128 \\
\hline Tempo de uso nos domingos, min & $0(0-720)$ & $0(0-240)$ & 0,028 \\
\hline Tempo de uso na semana, dias & $0(0-7)$ & $(0-7)$ & 0,655 \\
\hline \multicolumn{4}{|l|}{ MG } \\
\hline Uso do MG & $1(2)$ & $1(2)$ & 1,0 \\
\hline Uso do MG no dia anterior & $1(2)$ & $1(2)$ & 1,0 \\
\hline Tempo de uso na semana, dias & $0(0-5)$ & $0(0-2)$ & 0,775 \\
\hline Tempo de uso no dia anterior, min & $0(0-15)$ & $0(0-2)$ & 0,713 \\
\hline
\end{tabular}

Os resultados são apresentados em n (\%) e mediana (variação), min - minutos. 


\section{Discussão}

Este foi o primeiro estudo que avaliou extensivamente alterações do aparelho locomotor e uso de computador e videogame em adolescentes obesos e eutróficos. Evidenciou uma alta freqüência de dor músculoesquelética em ambos os grupos. Assim como, foram observadas uma baixa utilização do computador e minigame e presença de alterações ortopédicas localizadas predominantemente nos obesos. $O$ gênero feminino foi mais prevalente em obesos que apresentavam dor músculo-esquelética.

Uma das grandes vantagens do presente estudo foi a utilização de um questionário auto-aplicável, com perguntas objetivas e que havia sido previamente idealizado e utilizado em adolescentes da cidade São Paulo pelo grupo de Reumatologia do Instituto da Criança $(\mathrm{ICr})^{21,23,24}$. Este instrumento incluía também uma figura do corpo para o adolescente localizar respectivamente a dor referida, conforme utilizado também por outros autores $^{30}$. Para reduzir o viés de memória, utilizou-se como período analisado a presença do sintoma músculo-esquelético nos últimos três meses anteriores ao estudo, diferentemente de outros autores que usaram o período de um ano ${ }^{14}$. Outro aspecto relevante foi um alto índice de Kappa entre o pré-teste e a sua replicação ${ }^{31}$, demonstrando excelente confiabilidade das respostas nesse grupo de adolescentes de classe média brasileira. Essa análise é uma medida estatística de intensidade de 
concordância inter-observador ${ }^{31}$.

O presente estudo demonstrou uma alta prevalência de dor músculoesquelética que foi referida igualmente em adolescentes obesos e eutróficos, principalmente dor em membros inferiores e dor no músculo trapézio. Uma freqüência de $40 \%$ de dor musculoesquelética em adolescentes eutróficos foi também observada em outro estudo do grupo de Reumatologia do $\mathrm{ICr}^{23}$. Nos Estados Unidos, $61 \%$ de 135 crianças e adolescentes obesos referiam pelo menos uma dor músculo-esquelética, não existindo nesse estudo um grupo controle saudável ${ }^{10}$. Dor torácica foi mais frequentemente referida nos adolescentes eutróficos. Entretanto não foram evidenciadas alterações estruturais no exame físico geral ou no específico, como por exemplo síndrome miofascial.

A dor referida pelos adolescentes obesos e eutróficos deve ter sido preferencialmente aguda, transitória e localizada, e pode também ter sido superestimada por eles. De fato, o exame físico que confirmou as síndromes e/ou lesões músculo-esqueléticas e que habitualmente incluem doenças dolorosas crônicas, evidenciou tais alterações em apenas 18\% dos obesos que tinham dor. Dor crônica, ou seja, dor com duração acima de três meses, foi apenas observada em um adolescente eutrófico com fibromialgia juvenil.

Outro aspecto interessante foi que as alterações ortopédicas localizadas, principalmente encurtamento de quadríceps e geno valgo, foram evidenciadas em adolescentes obesos assim como reportadas em outros estudos $^{11-13}$, Muitos desses adolescentes (44\%) referiam dor músculo- 
esquelética que deverá se intensificar com a manutenção da obesidade ao longo da vida, determinando alterações biomecânicas no sistema locomotor $^{7}$. A persistência da obesidade nos adultos poderá causar uma elevação da força de sustentação, ocasionando osteoartrite com erosões em joelhos e quadris ${ }^{8,12}$, assim como um maior risco de fraturas ${ }^{11}$, dificuldades de movimento ${ }^{32}$, epifisiólise ${ }^{11}$ e tíbia vara ${ }^{8}$. Essas alterações ortopédicas ao proporcionar maior adesão ao sedentarismo tenderiam a perpetuar o ganho de peso anormal nesses pacientes ${ }^{6}$.

A exposição dos jovens aos aparelhos eletrônicos é um fenômeno crescente no mundo ${ }^{21,33-35}$ em todas as classes sócio-econômicas ${ }^{36}$, como evidenciado no presente estudo. Ressalte-se que, o computador e o minigame foram utilizados com menor frequência pelos obesos, e apenas o maior tempo de uso do videogame no domingo foi associado com dor nesses pacientes. Esse aspecto também foi reportado em um estudo espanhol evidenciando o excesso de peso como explicação pela utilização de videogame durante o final de semana ${ }^{37}$.

O único fator demográfico associado à presença de dor músculoesquelética em adolescentes obesos foi o sexo feminino, concordando com outros estudos $^{16,20,23}$. Na literatura médica o gênero feminino apresenta diferenças na percepção da dor, na forma de se relacionar com ela e, possivelmente, em seu maior relato. Além disto, o sexo feminino apresenta um limiar mais baixo, uma menor tolerância à dor ${ }^{23}$ e uma maior prevalência dessa em todas as faixas etárias ${ }^{16,20,23}$. 
Esse presente estudo tem limitações, pois não incluiu avaliação psicológica dos adolescentes. Distúrbios emocionais podem ser causa de dor ${ }^{20}$ e são fatores associados indiscutivelmente à obesidade na adolescência $^{2,8}$. Deverá ser realizado um futuro estudo nessa mesma população, a partir de análise radiográfica padronizada (avaliações do alinhamento das epífises, metáfises e diáfises dos membros inferiores), estadios puberais e das complicações metabólicas da obesidade.

Os pacientes obesos desse estudo apresentaram hipertensão arterial sistêmica (HAS), elevados valores de IMC e de circunferência abdominal, conforme amplamente é reportado na literatura ${ }^{1,24}$. Entretanto, o peso excessivo não parece ter contribuído para a presença de queixas de dor músculo-esquelética. É importante ressaltar que a população estudada esteve predominantemente no início da adolescência e que a obesidade crônica nesses jovens adolescentes pode determinar futuramente alterações ósteo-articulares e de partes moles irreversíveis.

Um programa de atividade física supervisionada ou em grupo (como natação, ciclismo, corrida, caminhada, etc) pode melhorar a dor, propiciar um fortalecimento muscular, melhorar a autoestima e a qualidade de vida, conforme evidenciado em outras doenças crônicas pediátricas ${ }^{38,39}$. Orientações de ergonomia e relaxamento muscular devam ser ensinadas no ambiente escolar dos adolescentes e aos seus professores ${ }^{24,40}$.

Adolescentes obesas do sexo feminino relataram mais dor músculoesquelética, sugerindo que programas específicos para esse gênero 
precisam ser desenvolvidos. Obesidade pode causar danos ao sistema ósteo-articular no início da adolescência, principalmente nos membros inferiores. Estudos longitudinais para avaliar o impacto da obesidade na biomecânica músculo-esquelética dos adolescentes serão necessários. 


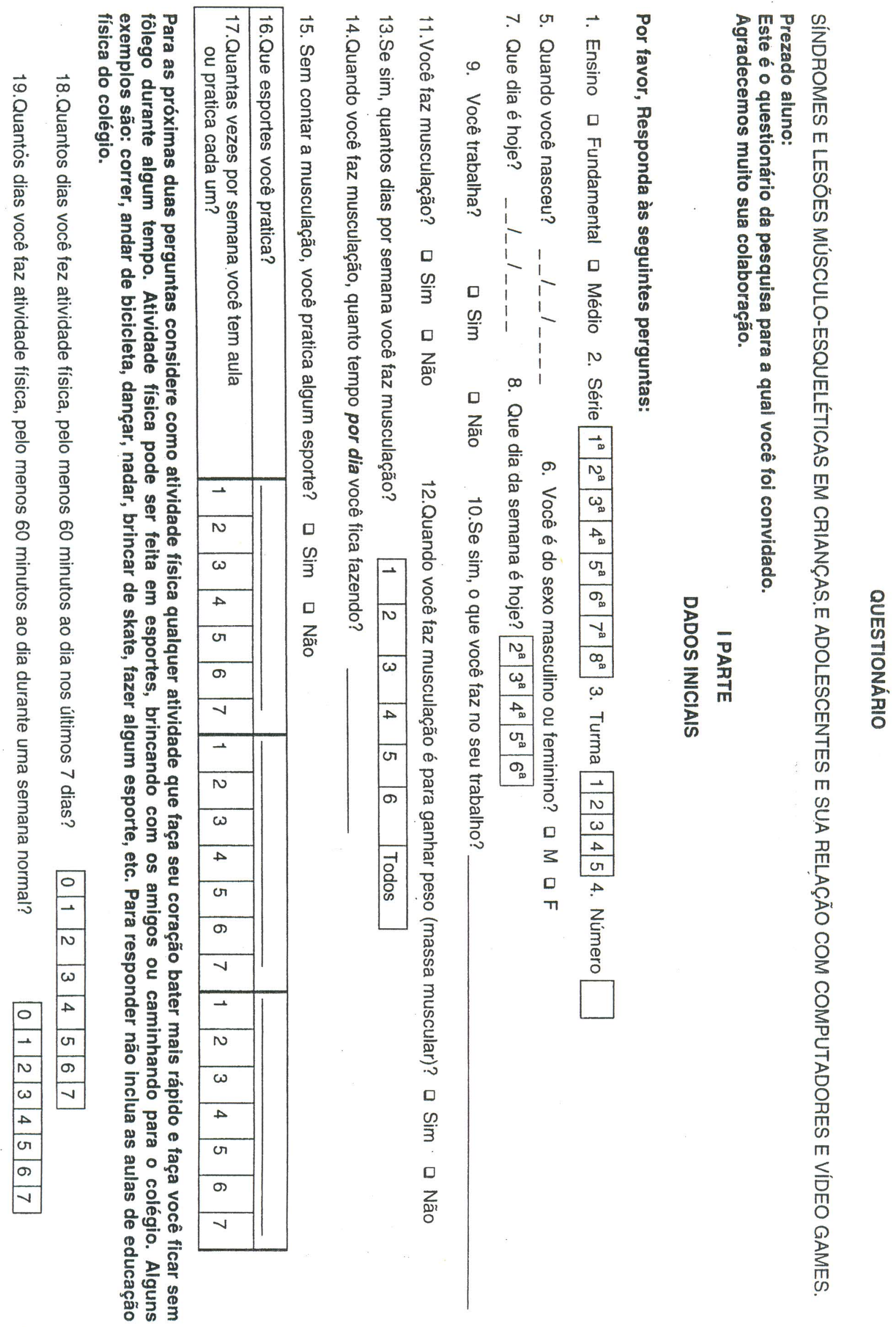




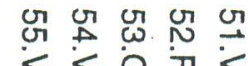

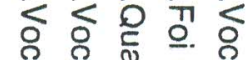

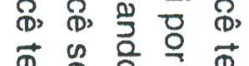
उ 응 蛋 모에 కై

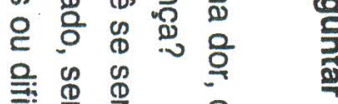

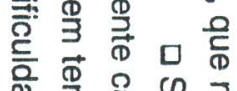

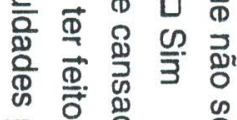

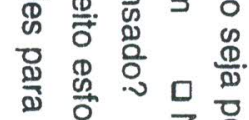
윽 응 귱유 光. 弯 는

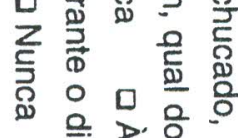

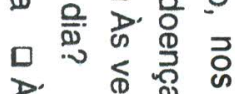

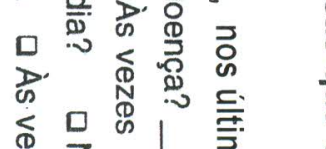

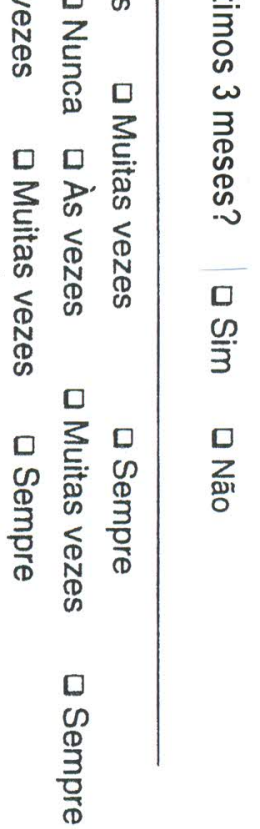

ㅇํำ

离

응

क्ञ

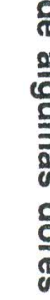

品
足

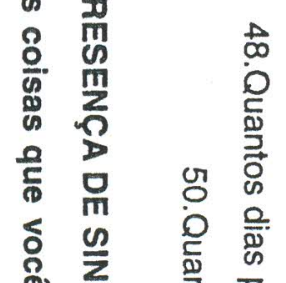

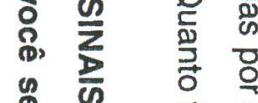

急只

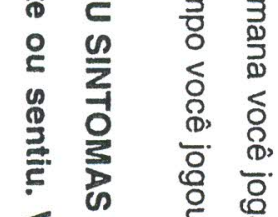

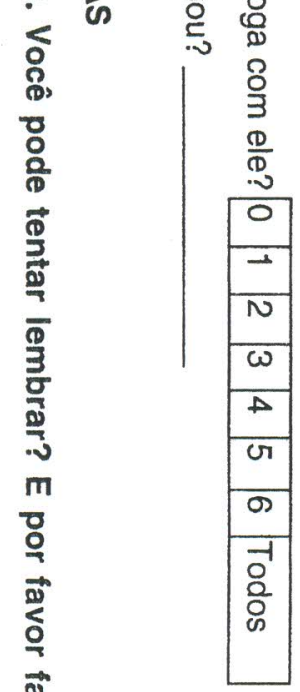

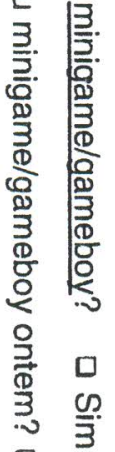

?

3$$
\text { ( }
$$

के $\vec{A} \pm \overrightarrow{0}$ W

<

Ф. क.

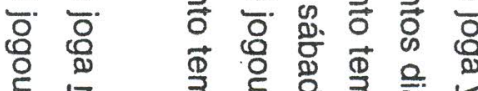

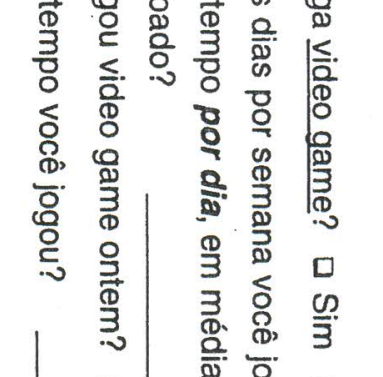

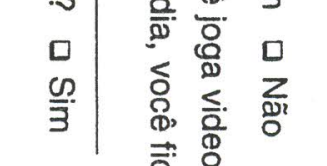
ते व

离.

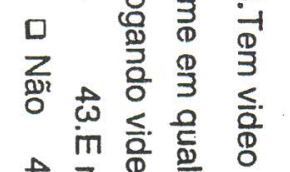

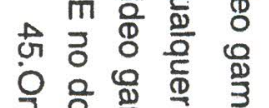

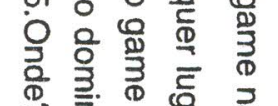

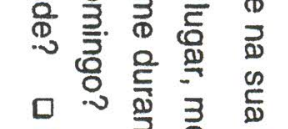

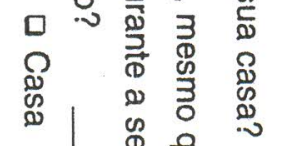

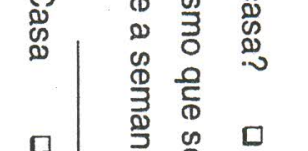

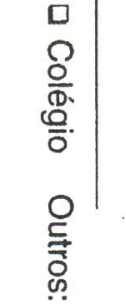

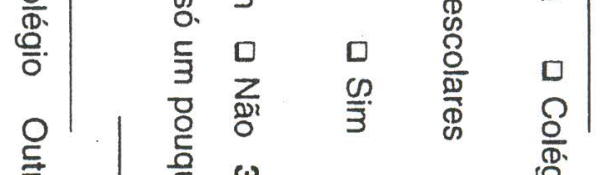

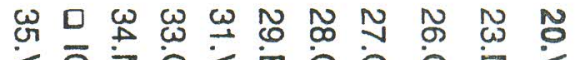

く๐ู

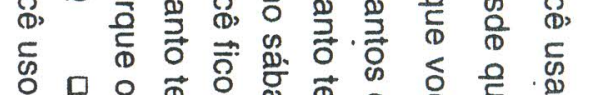

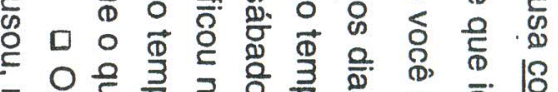

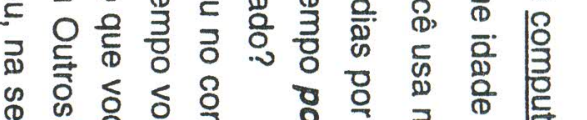

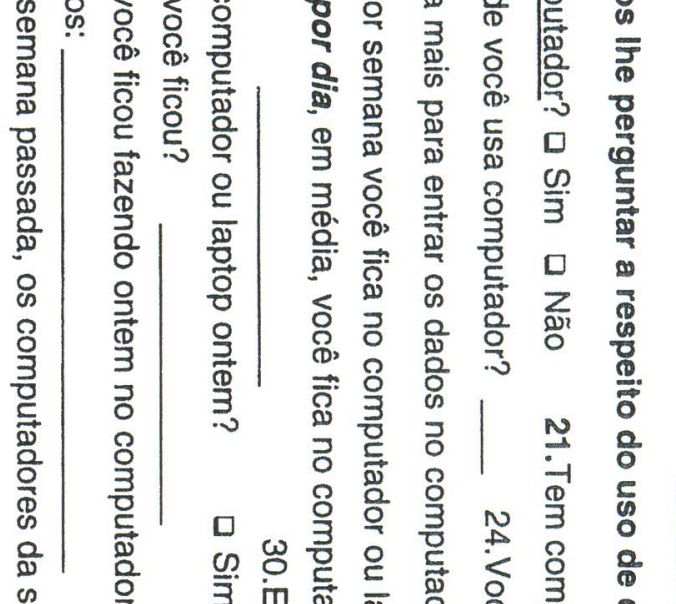

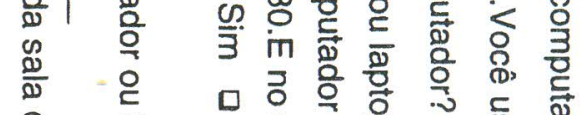

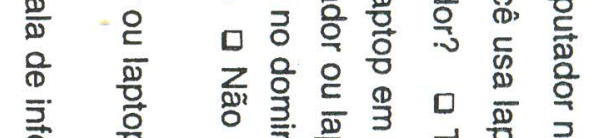

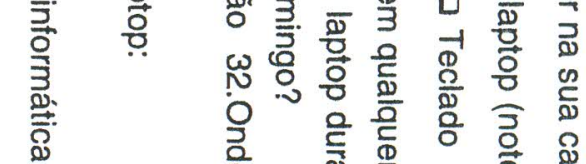

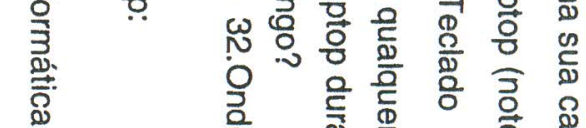

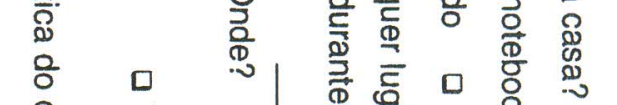

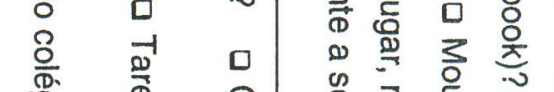

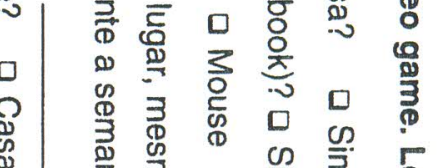

응

8

正

문

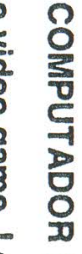

익

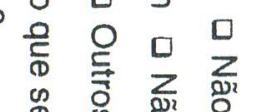

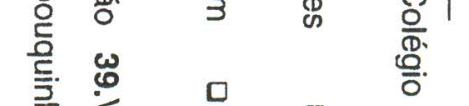

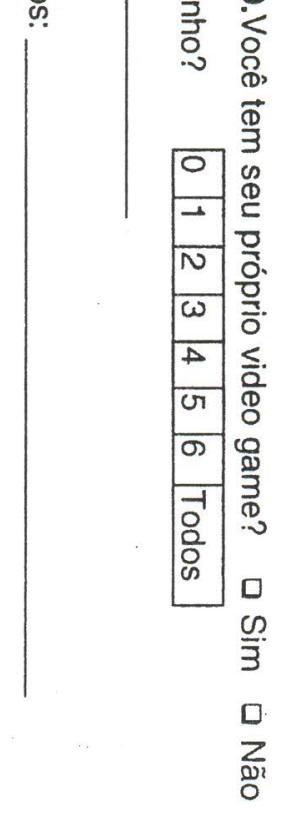

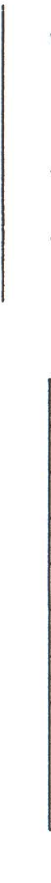

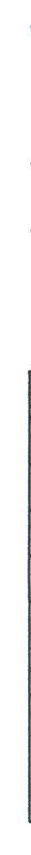

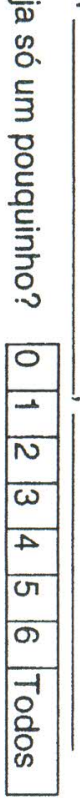

柋等

핑

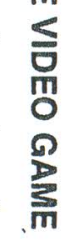

ธ

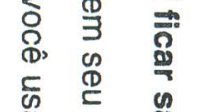

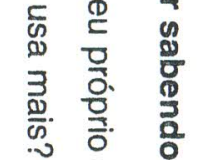

每

ㅁํㅇํำ

ᄋ 흔

공

둥 잉 


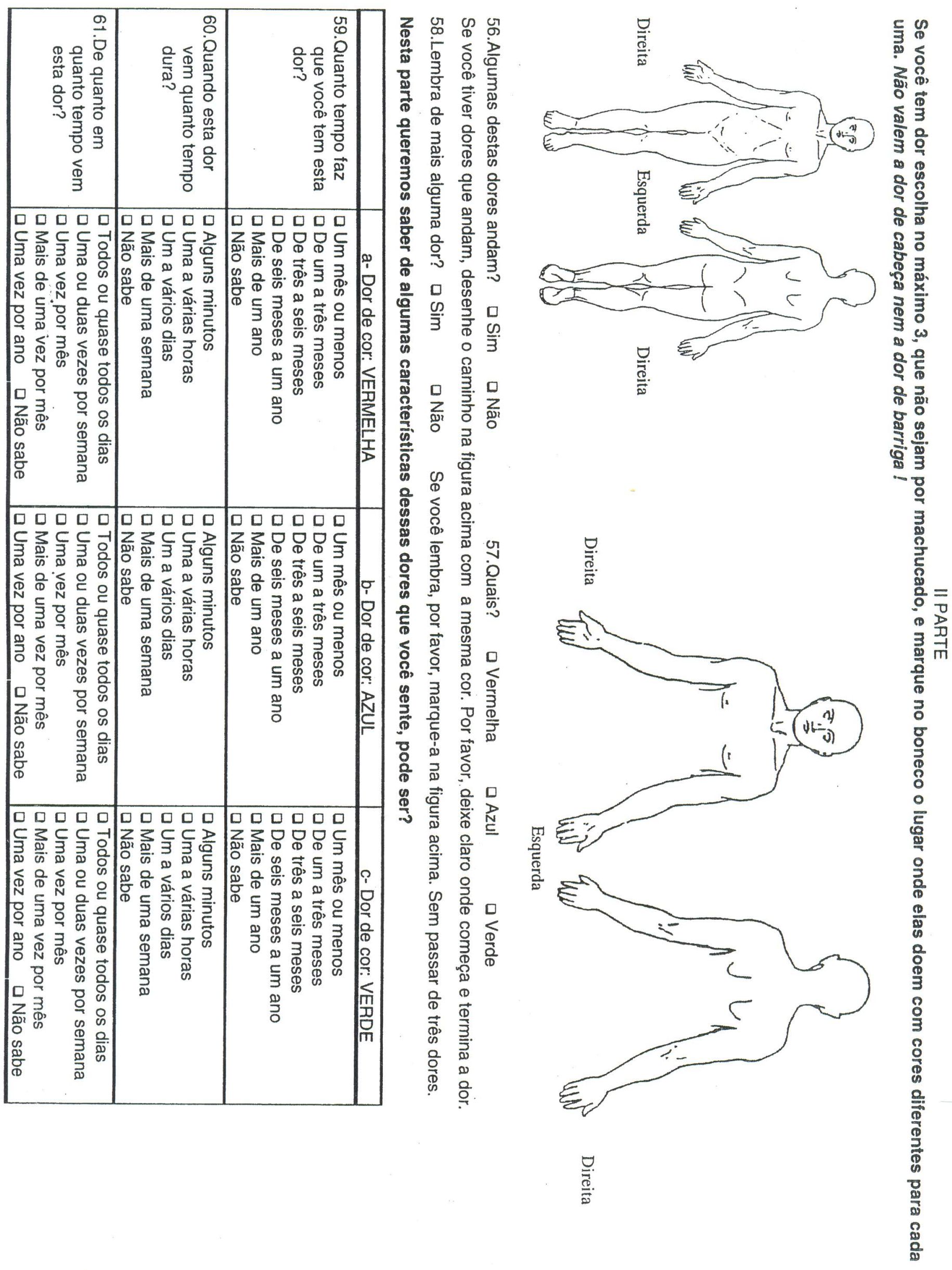




\begin{tabular}{|c|c|c|c|c|c|c|c|c|c|c|c|c|c|}
\hline 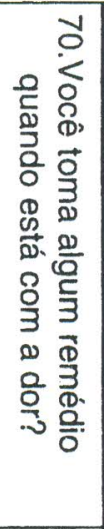 & & 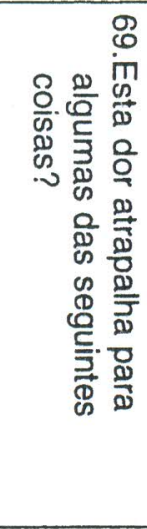 & 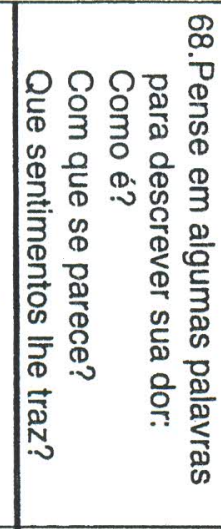 & 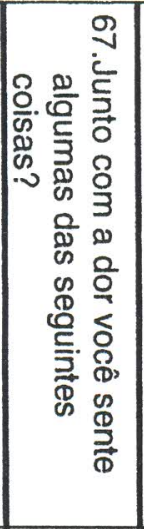 & 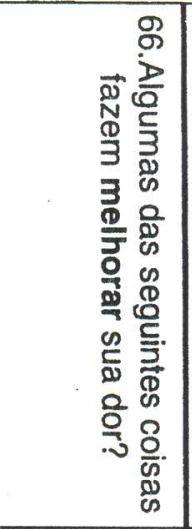 & 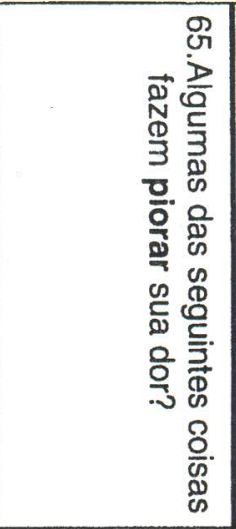 & 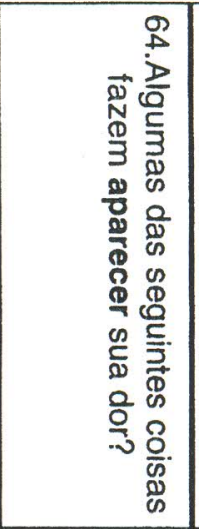 & & 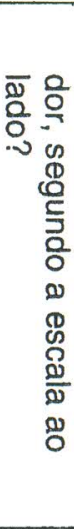 & & & 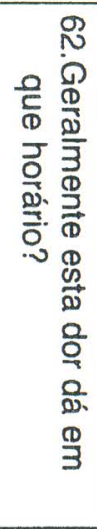 & \\
\hline 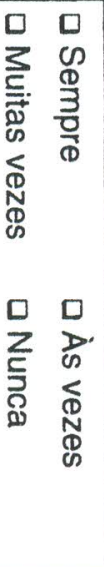 & & 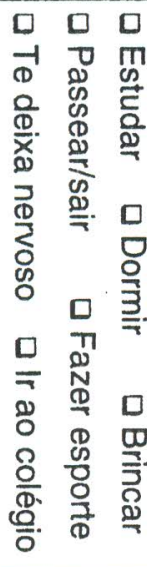 & & 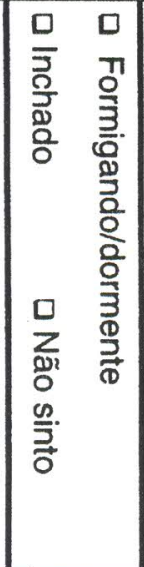 & 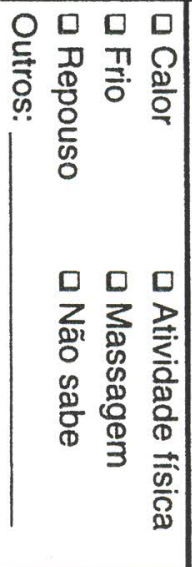 & 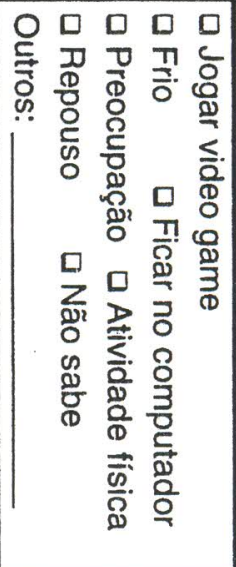 & 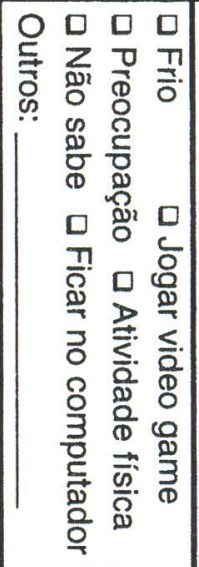 & $\begin{array}{l} \\
n \\
\infty \\
\frac{\Phi}{3} \\
00 \\
0 \\
0 \\
0 \\
-1\end{array}$ & 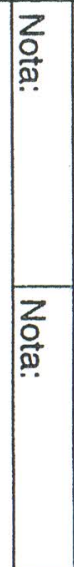 & $\begin{array}{l}\mathbb{Q} \\
\mathbb{D} \\
\underline{\omega} \\
\overline{3} \\
\mathbb{\Phi} \\
\overrightarrow{\mathbb{D}}\end{array}$ & 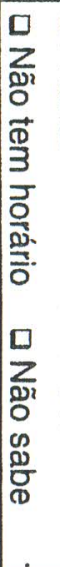 & 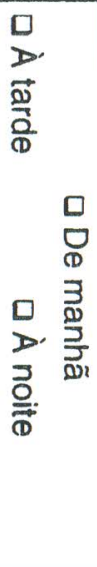 & 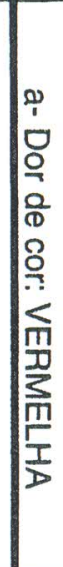 \\
\hline 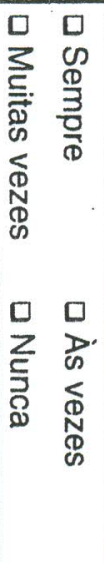 & 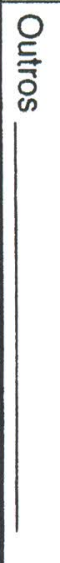 & 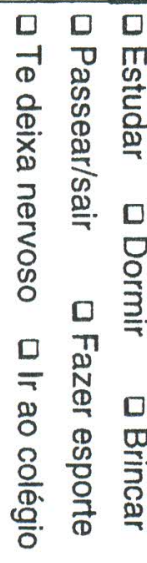 & & 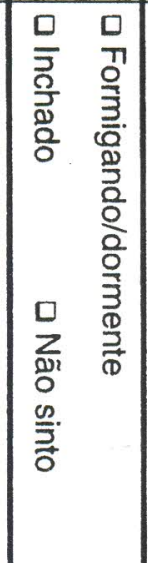 & 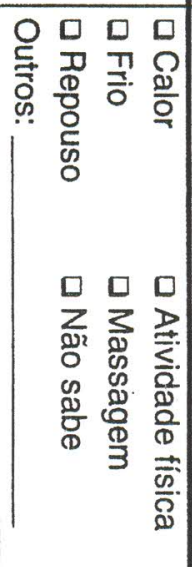 & 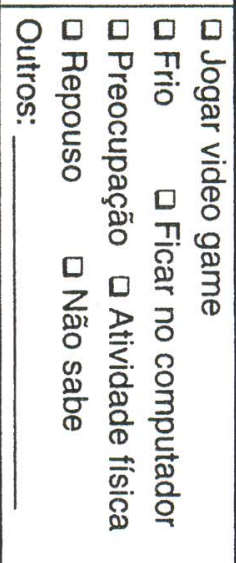 & 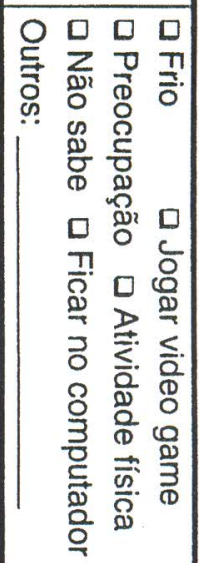 & $\begin{array}{l}\sigma \\
\sigma \\
-V\end{array}$ & 艿 & 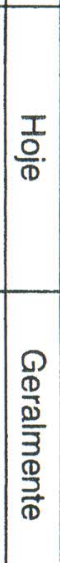 & 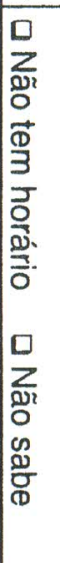 & 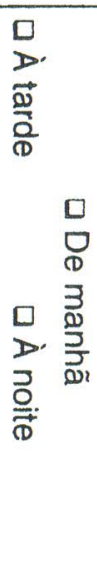 & 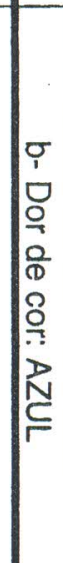 \\
\hline 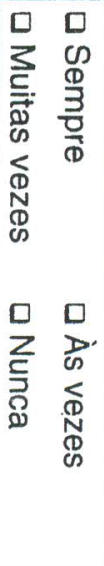 & 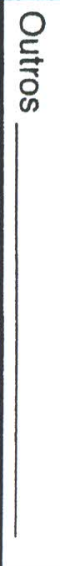 & 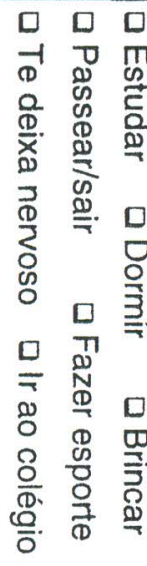 & & 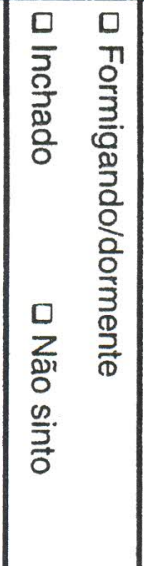 & 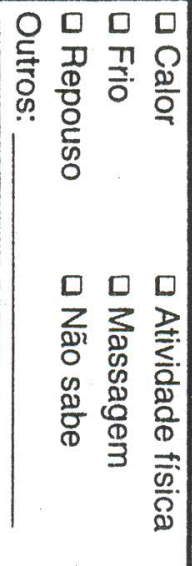 & 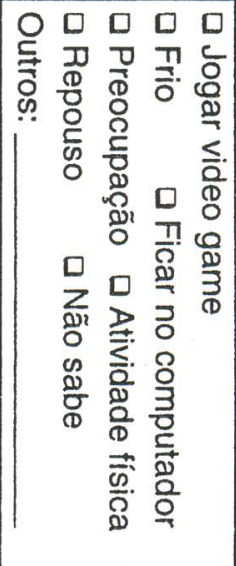 & 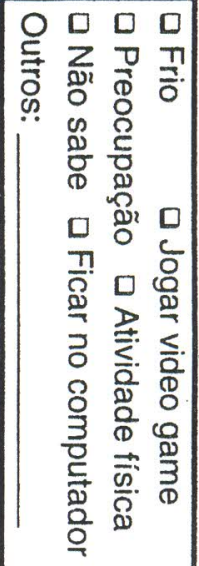 & 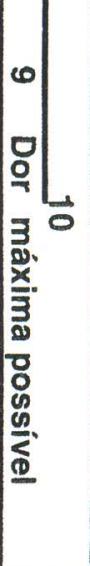 & 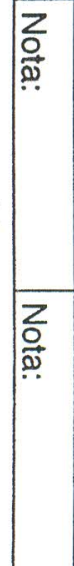 & 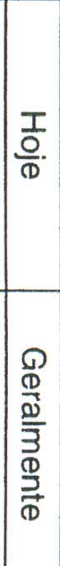 & 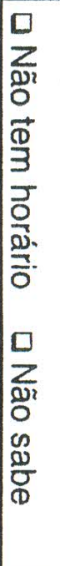 & 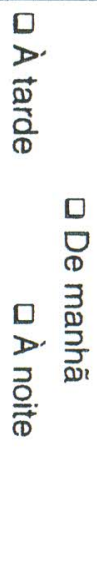 & 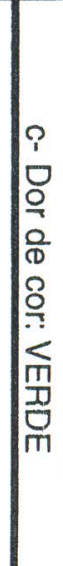 \\
\hline
\end{tabular}


CONCLUSÕES 


\section{Conclusões}

1. Dor músculo-esquelética referida foi prevalente em adolescentes obesos e eutróficos, em contraste com uma menor frequência das síndromes dolorosas.

2. Alterações ortopédicas localizadas foram observadas predominantemente nos obesos.

3. Uma baixa utilização do computador e do minigame foi evidenciada nos adolescentes obesos.

4. Adolescentes obesas do sexo feminino relataram mais dor músculo-esquelética, 


\section{Referências}

1. Catenacci VA, Hill JO, Wyatt HR. The obesity epidemic. Clin Chest Med $2009 ; 0: 415-44$.

2. Bruni V, Dei M, Peruzzi E, Seravalli V. The anorectic and obese adolescent. Best Pract Res Clin Obstet Gynaecol 2010;24:243-58.

3. Enes CC, Slater B. Obesity in adolescence and its main determinants. Rev Bras Epidemiol 2010;13:163-71.

4. In-Iw S, Biro FM. Adolescent Women and Obesity. J Pediatr Adolesc Gynecol 2011;24:58-61.

5. Wang Y, Monteiro C, Popkin BM. Trends of obesity and underweight in older children and adolescents in the United States, Brazil, China, and Russia. Am J Clin Nutr 2002;75:971-7.

6. Taylor ED, Theim KR, Mirch MC, Ghorbani S, Tanofsky-Kraff M, AdlerWailes DC, et al. Orthopedic complications of overweight in children and adolescents. Pediatrics 2006;117:2167-74.

7. Wearing SC, Hennig EM, Byrne NM, Steele JR, Hills AP. Musculoskeletal disorders associated with obesity: a biomechanical perspective. Obes Rev 2006;7:239-50.

8. Daniels SR. The consequences of childhood overweight and obesity. Future Child 2006;16:47-67. 
9. de Sá Pinto AL, de Barros Holanda PM, Radu AS, Villares SM, Lima FR. Musculoskeletal findings in obese children. J Paediatr Child Health 2006;42:341-4.

10.Stovitz SD, Pardee PE, Vazquez G, Duval S, Schwimmer JB. Musculoskeletal pain in obese children and adolescents. Acta Paediatr 2008;97:489-93.

11. Chan G, Chen CT. Musculoskeletal effects of obesity. Curr Opin Pediatr $2009 ; 21: 65-70$.

12. Ells LJ, Lang R, Shield JP, Wilkinson JR, Lidstone JS, Coulton S, et al. Obesity and disability - a short review. Obes Rev 2006;7:341-5.

13. Gettys FK, Jackson JB, Frick SL. Obesity in pediatric orthopaedics. Orthop Clin North Am. 2011;42:95-105.

14.Fassa AG, Facchini LA, Dall'Agnol MM, Christiani DC. Child labor and musculoskeletal disorders: the Pelotas (Brazil) epidemiological survey. Public Health Rep 2005;120:665-73.

15. Boström M, Dellve L, Thomée S, Hagberg M. Risk factors for generally reduced productivity-a prospective cohort study of young adults with neck or upper-extremity musculoskeletal symptoms. Scand J Work Environ Health 2008;34:120-32.

16.Grimby-Ekman A, Andersson EM, Hagberg M. Analyzing musculoskeletal neck pain, measured as present pain and periods of pain, with three different regression models: a cohort study. BMC Musculoskelet Disord 2009;10:73. 
17. Smith L, Louw Q, Crous L, Grimmer-Somers K. Prevalence of neck pain and headaches: impact of computer use and other associative factors. Cephalalgia 2009;29:250-7.

18. Jacobs K, Hudak S, McGiffert J. Computer-related posture and musculoskeletal discomfort in middle school students. Work 2009;32:275-83.

19. Kelly G, Dockrell S, Galvin R. Computer use in school: its effect on posture and discomfort in schoolchildren. Work 2009;32:321-8.

20. Diepenmaat AC, van der Wal MF, de Vet HC, Hirasing RA. Neck/shoulder, low back, and arm pain in relation to computer use, physical activity, stress, and depression among Dutch adolescents. Pediatrics 2006;117:412-6.

21.Zapata AL, Moares AJ, Leone C, Doria-Filho U, Silva CA. Pain and musculoskeletal pain syndromes related to computer and video game use in adolescents. Eur J Pediatr 2006;165:408-14.

22. Must A, Dallal GE, Dietz WH. Reference data for obesity: 85th and 95th percentiles of body mass index (wt/ht2) and triceps skinfold thickness. Am J Clin Nutr 1991;53:839-46.

23.Zapata AL, Moraes AJP, Leone C, Doria-Filho U, Silva CA. Pain and musculoskeletal pain syndromes in adolescents. J Adolesc Health 2006; 38:769-71.

24.Silva CA, Zapatta AL, Moares AJ, Doria-Filho U, Leone C. Utilização do computador e de jogos eletrônicos e avaliação da ergonomia com uso do computador em adolescentes de uma escola privada na cidade de São Paulo. Rev Paul Pediatria 2006;24:104-10. 
25. Almeida PM, Wickerrhauser H. Critério de classe econômica da Associação Brasileira de Anunciantes (ABA) e Associação Brasileira dos Institutos de Pesquisa de Mercado (ABIPEME) 1991, p 1-29.

26. National High Blood Pressure Education Program Working Group on High Blood Pressure in Children and Adolescents. The fourth report on the diagnosis, evaluation, and treatment of high blood pressure in children and adolescents. Pediatrics 2004;114:555-76.

27. Hoppenfeld S, Hutton R. Physical examination of the spine \& extremities. In: Hoppenfeld S, Hutton R, eds. Physical Examination of the Knee, Ankle,Foot and Lumbar Spine. New York: Prentice Hall, 1976:171-264.

28. Wolfe F, Smythe HA, Yunus MB, Bennett RM, Bombardier C, Goldenberg DL, et al. The American College of Rheumatology 1990 criteria for the classification of fibromyalgia. Report of multicentre criteria committee. Arthritis Rheum 1990;33:160-72.

29. Beighton P, Solomon L, Soskolne CL. Articular mobility in an African population. Ann Rheum Dis 1973;32:413-8.

30. Mikkelsson M, Salminen JJ, Kautiainen H. Non-specific musculoskeletal pain in preadolescents: prevalence and 1-year persistence. Pain 1997:73:29-35.

31.Landis JR, Koch GG. The measurement of observer agreement for categorical data. Biometrics 1977;33:159-74.

32. Tsuritani I, Honda R, Noborisaka Y, Ishida M, Ishizaki M, Yamada Y. Impact of obesity on musculoskeletal pain and difficulty of daily movements in Japanese middle-aged women. Maturitas 2002;42:23-30. 
33. Van der Voort T, Beentjes WWJ, Bovill MI. Y. Young people's ownership and uses of new and old forms of media in Britain and The Netherlands. Eur $\mathrm{J}$ Commun 1998;13:457-77.

34. Johnsson-Smaragdi U, d'Haenens L, Krotz F. Patterns of old and new media use among young people in Flanders, Germany and Sweden. Eur J Commun 1998;13:479-501.

35. Roberts DF. Media and youth: access, exposure and privatization. J Adolesc Health. 2000;27:8-14.

36. Devís-Devís J, Peiró-Velert C, Beltrán-Carrillo VJ, Tomás JM. Screen media time usage of 12-16 year-old Spanish school adolescents: Effects of personal and socioeconomic factors, season and type of day. J Adolesc 2009;32:21331.

37. Vicente-Rodríguez G, Rey-López JP, Martín-Matillas M, Moreno LA, Wärnberg J, Redondo C, et al. Television watching, videogames, and excess of body fat in Spanish adolescents: the AVENA study. Nutrition 2008;24:65462.

38. Gualano B, Sá Pinto AL, Perondi B, Leite Prado DM, Omori C, Almeida RT, et al. Evidence for prescribing exercise as treatment in pediatric rheumatic diseases. Autoimmun Rev 2010;9:569-73.

39. Omori C, Prado DM, Gualano B, Sallum AM, Sá-Pinto AL, Roschel H, et al. Responsiveness to exercise training in juvenile dermatomyositis: a twin case study. BMC Musculoskelet Disord 2010;11:270. 
40. Campos LM, Silva CA. Como minimizar a má influência do computador. Rev Paulista Reumatol 2008;7:5-7. 
Artigo encaminhado ao Jornal de Pediatria 


\section{ARTIGO ORIGINAL}

Dor, síndromes músculo-esqueléticas e alterações ortopédicas em adolescentes obesos e uso de computador e videogames

\section{Pain, musculoskeletal syndromes and orthopedics abnormalities related to computer and video game use in obese adolescents}

Suely Nóbrega Jannini ${ }^{1}$, Ulysses Dória-Filho ${ }^{2}$, Durval Damiani ${ }^{2}$, Clovis Artur Almeida Silva ${ }^{1}$

1- Unidade de Reumatologia Pediátrica do Instituto da Criança do Hospital das Clinicas da Faculdade de Medicina da Universidade de São Paulo (ICr-HC-FMUSP), São Paulo - SP lattes.cnpq.br/6033796445954274

2- Núcleo de Consultoria e Apoio em Metodologia de Pesquisa e Estatística do Departamento de Pediatria da FMUSP lattes.cnpq.br/1893123519788586

3- Unidade de Endocrinologia Pediátrica do ICr-HC-FMUSP, São Paulo - SP - lattes.cnpq.br/0787781193947922

4- Unidade de Reumatologia Pediátrica e Disciplina de Reumatologia da FMUSP - Lattes.cnpq.br/4428785157940057

Não há conflitos de interesses.

\section{Endereço para correspondência:}

Prof. Dr. Clovis Artur Almeida Silva

Rua Araioses, 152/81- Vila Madalena - São Paulo - SP - Brasil

CEP - 05442-010 - (55 11) 3069-8563 - Fax: (55 11) 3069-8503

E-mail: clovis.silva@icr.usp.br 


\section{RESUMO}

Objetivo: Avaliar presença de dor, síndromes músculo-esqueléticas, alterações ortopédicas e uso de computador e videogame em adolescentes.

Métodos: Um estudo transversal avaliou 100 adolescentes consecutivos com obesidade e 100 eutróficos a partir de um questionário confidencial, auto-aplicável, incluindo dados demográficos, prática esportiva, sintomas dolorosos do sistema músculo-esquelético e uso de computador e videogame. Pré-teste e re-teste do questionário foram realizados. $\mathrm{O}$ exame físico avaliou seis síndromes músculo-esqueléticas e sete alterações ortopédicas.

Resultados: O índice de Kappa entre pré-teste e ré-teste foi de 0,724. Dor e síndromes músculo-esqueléticas foram igualmente prevalentes nos dois grupos ( $44 \%$ vs. $56 \%, p=0,09 ; 12 \%$ vs. $16 \%, p=0,541$; respectivamente). Entretanto, alterações ortopédicas ( $98 \%$ vs. $76 \%, p=0,0001$ ), encurtamento de quadríceps $(89 \%$ vs. $44 \%, p=0,0001)$ e geno valgo $(87 \%$ vs. $24 \%$, $\mathrm{p}=0,0001$ ) foram significativamente mais evidenciados nos obesos versus controles. As medianas do tempo de uso do computador no dia anterior a pesquisa, nos sábados e domingos foram menores nos obesos (30 vs. 60 minutos, $p=0,0001 ; 1$ vs. 60 minutos, $p=0,001 ; 0$ vs. 30 minutos, $p=0,02$; respectivamente). Uso de minigame foi menor nos obesos ( $2 \%$ vs. $11 \%$, $p=0,003$ ), não havendo diferença no uso de videogame nos dois grupos ( $p>0,05)$. Comparações entre obesos com e sem dor evidenciaram maior frequência no gênero feminino ( $59 \%$ vs. $39 \%, p=0,048)$ e maior mediana de tempo de uso nos domingos [0 (0-720) vs. $0(0-240)$ minutos, $p=0.028$ ].

Conclusões: Obesidade pode causar danos ao sistema ósteo-articular no início da adolescência, principalmente nos membros inferiores. Programas específicos para adolescentes obesos do sexo feminino com dor músculoesquelética precisam ser desenvolvidos.

Palavras-chave: adolescente, obesidade, dor, sindrome musculo-esqulética, computador, videogame. 


\section{ABSTRACT}

Objective: To evaluate the presence of pain, musculoskeletal syndromes and orthopedic abnormalities and the use of computers and videogames in adolescents.

Methods: A cross-sectional study using a self-applicable questionnaire to assess 100 obese adolescents and 100 healthy controls included demographic data, sports, musculoskeletal painful symptoms and the use of computer and videogames. A. pretest and its replication were performed. The physical examination evaluated six musculoskeletal syndromes and seven orthopedics alterations.

Results: The Kappa index between the pretest and its replication was 0.724 . Pain and musculoskeletal syndromes were similar in both groups (44\% vs. $56 \%, p=0.09 ; 12 \%$ vs. $16 \%, p=0.541$; respectively). However, orthopedics alterations $(98 \%$ vs. $76 \%, p=0.0001)$, tight quadriceps $(89 \%$ vs. $44 \%$, $\mathrm{p}=0.0001)$ and genu valgum ( $87 \%$ vs. $24 \%, \mathrm{p}=0.0001)$ were significantly observed in obese versus controls. The median time spent using computer on the prior to the research and on Saturdays and Sundays were lower in obese ( 30 vs. 60minutes, $p=0.0001 ; 1$ vs. 60minutes, $p=0.001 ; 0$ vs. 30 minutes, $p=0.02$; respectively). Minigame use was lower in obese ( $2 \%$ vs. $11 \%, p=0.003$ ), without any differences in the use of videogames in both groups ( $p>0.05$ ). Comparisons between obese adolescents with and without musculoskeletal pain demonstrated higher frequency in females $(59 \%$ vs. $39 \%, p=0.048$ ).

Conclusions: Obesity can induce damages to the osteoarticular system at the beginning of the adolescence, mainly in the inferior limbs. Specific programs for female obese adolescents with musculoskeletal pain should be established.

Key-words: adolescent, obesity, pain, musculoskeletal syndrome, computer, video game. 


\section{INTRODUÇÃO}

Obesidade e sobrepeso na adolescência são indiscutíveis problemas mundiais de saúde pública ${ }^{1-4}$, incluindo no Brasil. Wang et al., $2002^{5}$ avaliaram a prevalência de sobrepeso em quatro países (Brasil, Rússia, China e Estados Unidos da América) nas últimas décadas e constataram um incremento importante no Brasil de $240 \%$.

A obesidade na adolescência é uma doença crônica multifatorial e está relacionada a fatores genéticos ${ }^{4}$, mudanças dos padrões alimentares, redução da atividade física e incremento da utilização de computador e videogames, entre outros fatores ${ }^{3}$. Adolescentes obesos têm maior prevalência de co-morbidades e complicações, tais como: hipertensão arterial, dislipidemia, intolerância à glicose ${ }^{1,2,4}$, câncer, doenças pulmonares e dermatológicas, assim como: alterações gastrintestinais, gênito-urinárias e neuropsiquiátricas, entre outras ${ }^{1,2}$.

Alguns estudos evidenciaram que adolescentes obesos apresentam também uma maior prevalência de dor músculo-esquelética localizada ${ }^{6-11}$, principalmente em membros inferiores ${ }^{6,10,11}$ e em região lombar ${ }^{9,10-12}$; assim como alterações ortopédicas localizadas ${ }^{12,13}$, tais como: geno valgo, geno varo $^{11,13}$, geno recurvatum e encurtamento de quadríceps ${ }^{9}$. Síndromes músculo-esqueléticas que apresentam dor músculo esquelética crônica e difusa, como fibromialgia juvenil, foram raramente estudadas nos adolescentes obesos ${ }^{9}$.

Outros estudos também evidenciaram associação entre uso de computador e dor músculo-esquelética em adolescentes e jovens eutróficos $^{14}$, tais como: dor em membros superiores ${ }^{15}$, dor cervical ${ }^{15-17} \mathrm{e}$ sensação de desconforto músculo-esquelético ${ }^{18,19}$. Em contraste, outros não evidenciaram associação entre diversos tipos de dor com o uso de computador em adolescentes eutróficos ${ }^{20,21}$ e para o nosso conhecimento não há estudos avaliando esses aspectos em adolescentes obesos.

Ainda não há pesquisa que tenha estudado simultaneamente dor, síndromes músculo esqueléticas, alterações ortopédicas e uso de 
computador e videogame em adolescentes obesos versus eutróficos. Além disto, nenhum estudo avaliou sistematicamente a prevalência de síndrome de hipermobilidade articular benigna (SHAB), síndrome miofascial, tendinites, bursites e epicondilites em adolescentes com esta doença crônica.

Assim sendo, os objetivos do presente estudo foram determinar presença de dor, síndromes músculo-esqueléticas, alterações ortopédicas, uso de computador e vídeo-game em adolescentes obesos e eutróficos. Além disto, avaliar possível associação entre dados demográficos, síndromes, alterações ortopédicas e uso de computador e videogame entre adolescentes obesos com dor versus adolescentes obesos sem dor músculo-esqueléticas.

\section{MÉTODOS}

\section{População do estudo}

Duzentos adolescentes consecutivos (100 adolescentes com obesidade e 100 eutróficos) do Ambulatório de Adolescentes do Hospital Regional Hans Dieter Schmidt (HRHDS), Joinville, Santa Catarina, foram avaliados durante o período de 2008 a 2010. O estudo foi aprovado pelas Comissões de Pesquisa e Ética do HRHDS e do Hospital das Clinicas da Faculdade de Medicina da Universidade de São Paulo (HC-FMUSP). Todos os adolescentes obesos e eutróficos, assim como os seus respectivos familiares, assinaram o termo de consentimento livre e esclarecido.

Obesidade foi definida de acordo com o índice de massa corpórea (IMC) maior ou igual ao percentil 95 National Control Health System (NCHS) de 2000 para sexo e idade. Eutrófico foi definido quando o IMC fosse menor ou igual ao percentil 85 e maior que o percentil 5 do NCHS para sexo e idade $^{22}$.

Os critérios de inclusão foram: adolescentes (idades entre 10 e 19 anos e 11 meses) e aceitação da participação do estudo pelos adolescentes e pais ou responsáveis legais. Os pacientes com dor e lesões músculo 
esqueléticos secundários as seguintes doenças foram excluídos: do colágeno, infecciosas, onco-hematológicas, genéticas, endócrinas (como doenças tireoidianas e diabetes mellitus) e traumáticas recentes. Além disto, os pacientes que utilizavam medicamentos (tais como glicocorticoides, anticonvulsivantes e anti-psicóticos) e os que tinham qualidade inadequada de preenchimento do questionário foram também excluídos.

\section{Metodologia}

A pesquisa consistiu de um estudo transversal e incluiu aplicação de um questionário e de exame físico geral e específico do sistema músculoesquelético por um profissional treinado.

O questionário foi confidencial, auto-aplicável e incluiu os seguintes aspectos: dados demográficos, prática esportiva, sintomas dolorosos do sistema músculo-esquelético presentes nos últimos três meses e questões relacionadas ao uso de computador e videogame ${ }^{21,23,24}$. As classes socioeconômicas das famílias em que os adolescentes estavam inseridos foram avaliadas de acordo a com a classificação da Associação Brasileira dos Institutos de Pesquisa de Mercados ${ }^{25}$.

Entre agosto e setembro de 2008, um pré-teste e um re-teste do questionário foram realizados em 30 adolescentes consecutivos (15 obesos e 15 eutróficos) com intervalo de 10 dias para avaliar a confiabilidade das respostas.

O questionário incluiu 70 questões e uma figura do corpo para localizar a respectiva dor, conforme utilizado e publicado anteriormente ${ }^{21,23,24}$. Dor músculo-esquelética foi definida como aqueles que responderam "sim" à presença de dor em qualquer localização nos últimos três meses da pesquisa. Os seguintes tipos de dor músculo-esquelética foram também sistematicamente avaliados: dor cervical, dor em membros superiores, dor torácica, dor em músculo trapézio, dor lombar e dor localizada em membros inferiores ${ }^{23}$. Com relação à utilização do computador, o questionário avaliou os seguintes aspectos: uso do computador, disponibilidade domiciliar, aparelho próprio, utilização de aparelho portátil (laptop), emprego de teclado, mouse ou outro. Além disto, 
incluiu avaliação de: idade de início do uso do computador em anos, tempo de uso deste em dias por semana, tempo de uso no dia anterior a pesquisa, tempo de uso nos sábados e nos domingos. O mesmo foi relatado em relação à utilização de videogame e minigame/gameboy ${ }^{21}$.

O exame físico geral incluiu avaliação do índice de massa corpórea (IMC - peso em quilograma dividido por altura ao quadrado, $\mathrm{Kg} / \mathrm{m}^{2}$ ) e avaliação da circunferência abdominal (em centímetros). As pressões arteriais sistólicas (PAS) e diastólicas (PAD) foram aferidas. Hipertensão arterial sistêmica foi definida como PAS e/ou PAD superior ou igual ao percentil 95 para o sexo, idade e estatura ${ }^{26}$.

Com relação ao exame músculo-esquelético, as seguintes síndromes músculo-esqueléticas foram avaliadas: fibromialgia juvenil, síndrome de hipermobilidade articular benigna (SHAB), síndrome miofascial, tendinites, bursites e epicondilites. Avaliação ortopédica da coluna e dos membros inferiores foi realizada para pesquisa de: escoliose, geno valgo, geno varo, geno recurvatum, encurtamento de quadríceps, encurtamento de isquiotibiais e hálux valgo ${ }^{27}$.

A fibromialgia juvenil foi diagnosticada segundo os critérios do Colégio Americano de Reumatologia pela demonstração de 11 dos 18 pontos dolorosos, associadas à presença de dor difusa, com duração mínima de três meses ${ }^{28}$. Hipermobilidade articular (HA) foi determinada de acordo com os critérios de Beighton. A SHAB foi definida como a presença de HA associada à dor no sistema músculo-esquelético e presença de 5 dos 9 critérios ${ }^{29}$. A síndrome miofascial foi diagnosticada pela presença de ponto gatilho ativo, definido como um ponto doloroso localizado em uma banda tensa muscular. Ao ser pressionado, esse induzia uma dor referida em áreas padronizadas e reprodutíveis para cada músculo ${ }^{23}$. A atividade do ponto gatilho foi definida pela confirmação do adolescente da existência anterior dessa dor. Estes foram avaliados nos grupos musculares acometidos em lesões por esforços repetitivos e nos que participam ativamente dos movimentos necessários para uso de computador e videogame. Os seguintes músculos ou grupos musculares foram avaliados bilateralmente: 
trapézio, subescapular, musculatura cervical posterior, bíceps, tríceps, braquioradial, musculatura extensora e flexora da mão, punho e dedos e musculatura intrínseca da mão ${ }^{23}$.

Além disto, com relação ao exame esquelético, com o paciente em posição supina, encurtamento de isquiotibiais foi avaliado com 0 posicionamento do quadril a $90^{\circ}$ e extensão do joelho, sendo observado o encurtamento dos isquiotibiais se a extensão do joelho fosse inferior a $15^{\circ}$. Com o paciente em pronação, a flexibilidade do quadríceps foi avaliada com o joelho completamente estendido, com a face anterior da coxa tocando a superfície de contato. O quadríceps era encurtado se os calcâneos não atingissem as nádegas ${ }^{27}$.

\section{Análise estatística}

Os resultados foram apresentados em mediana (variação) ou média \pm desvio-padrão para variáveis contínuas e número (\%) para variáveis categóricas. Para avaliar a confiabilidade do questionário entre o pré-teste e o re-teste foi utilizado o índice de Kappa. As medianas dos resultados foram comparadas pelo teste de Mann-Whitney para variáveis contínuas para determinar diferenças entre adolescentes obesos versus eutróficos e entre adolescentes obesos com dor versus adolescentes obesos sem dor. Para as variáveis categóricas, as diferenças foram calculadas pelo teste exato de Fisher e qui-quadrado. Valores de $p<0,05$ foram considerados estatisticamente significantes.

\section{RESULTADOS}

O índice de Kappa entre o pré-teste e re-teste foi de 0,724, demonstrando uma excelente confiabilidade nas respostas dos adolescentes obesos e eutróficos.

\section{Adolescentes obesos versus eutróficos}

As medianas do IMC e da circunferência abdominal foram 
significativamente maiores em adolescentes obesos versus eutróficos [29,45 $(23,7-45,4)$ vs. $18,8(14,8-24,1) \mathrm{kg} / \mathrm{m}^{2}, \mathrm{p}=0,0001 ; 95$ (69,5-123) vs. 69 (5583) $\mathrm{cm}, p=0,0001$; respectivamente]. Além disto, as medianas das pressões arterial sistólica e diastólica foram significativamente maiores em adolescentes obesos e eutróficos [110 (80-140) vs. 100 (70-130) mmHg $, \mathrm{p}=0,0001 ; 70$ (50-90) vs. 60 (40-80) $\mathrm{mmHg}, \mathrm{p}=0,0001$; respectivamente]. Não houve diferença estatística entre idade atual, gênero feminino, classes sócio-econômicas $\mathrm{C}$ e $\mathrm{D}$ e prática esportiva (Tabela 1).

As presenças de pelo menos uma dor músculo-esquelética e de pelo menos uma síndrome músculo-esquelética foram semelhantes em pacientes obesos versus eutróficos ( $44 \%$ vs. $56 \%, p=0,09 ; 12 \%$ vs. $16 \%, p=0,541$; respectivamente). Dor torácica e epicondilite foram significativamente menores em obesos versus controles ( $2 \%$ vs. $13 \%, p=0,005 ; 3 \%$ vs. $11 \%$, $\mathrm{p}=0,027$; respectivamente). Pelo menos uma alteração ortopédica, assim como encurtamento de quadríceps e geno valgo foram significativamente mais evidenciados nos obesos comparados aos controles $(98 \%$ vs. $76 \%$, $p=0,0001 ; 89 \%$ vs. $44 \%, p=0,0001 ; 87 \%$ vs. $24 \%, p=0,0001$; respectivamente] (Tabela 1 ).

As características do uso de computador, videogame e minigame em adolescentes obesos versus eutróficos estão na Tabela 2. As freqüências de disponibilidade domiciliar e do uso de computador no dia anterior a pesquisa foram significativamente menores nos obesos comparados aos eutróficos ( $54 \%$ vs. $68 \%, p=0,042$; $32 \%$ vs. $48 \%, p=0,015$; respectivamente). Além disto, as medianas da idade de início do uso, tempo de uso no dia anterior a pesquisa, tempo de uso no sábado e no domingo foram também significativamente menores nos obesos versus controles [10 (5-15) vs. 11 (516) anos, $p=0,01 ; 30$ (0-360) vs. 60 (0-720) minutos, $p=0,0001 ; 1$ (0-720) vs. 60 (0-600) minutos, $\mathrm{p}=0,001 ; 0$ (0-720) vs. 30 (0-720) minutos, $\mathrm{p}=0,02$; respectivamente]. Não houve diferença em relação ao uso de videogame e minigame nos dois grupos (Tabela 2). 


\section{Adolescentes obesos que referiam dor músculo-esquelética versus os sem dor}

Com relação às comparações entre adolescentes obesos que referiam dor músculo-esquelética versus os que não referiam, o gênero feminino teve mais freqüentemente dor ( $59 \%$ vs. $39 \%, p=0,048)$. Não houve diferença estatística entre idade atual, classes sócio-econômicas $C$ e $D$, IMC, circunferência abdominal, PA e prática esportiva nos dois grupos estudados (Tabela 3). Apesar de uma maior freqüência de síndromes músculo-esqueléticas nos adolescentes obesos com dor, não houve diferença entre esses e os que não referiam dor $(p>0,05)$. Quatro adolescentes obesos tinham tendinite/epicondilite e não referiram dor no questionário (Tabela 3). Outras alterações ortopédicas foram similares em adolescentes obesos com dor versus sem dor (Tabela 3).

Apenas a mediana do tempo de uso do vídeo-game no domingo foi significativamente maior em obesos que reportavam dor versus os obesos que não referiam dor [0 (0-720) vs. 0 minutos (0-240), $p=0,028]$ (Tabela 4). Não houve diferença significativa entre as outras variáveis do videogame, assim como do computador e minigame em obesos com dor versus obesos sem dor (Tabela 4).

\section{DISCUSSÃO}

Este foi o primeiro estudo que avaliou extensivamente alterações do aparelho locomotor e uso de computador e videogame em adolescentes obesos e eutróficos. Evidenciou uma alta freqüência de dor músculoesquelética em ambos os grupos. Assim como, foram observadas uma baixa utilização do computador e mini-game e alterações ortopédicas localizadas predominantemente nos obesos. O gênero feminino foi mais prevalente nos obesos com dor músculo-esquelética.

Uma das grandes vantagens do presente estudo foi a utilização de um questionário auto-aplicável, com perguntas objetivas e que havia sido previamente idealizado e utilizado em adolescentes da cidade São Paulo 
pelo nosso grupo $21,23,24$. Este instrumento incluía também uma figura do corpo para o adolescente localizar a respectiva dor referida, conforme utilizado também por outros autores ${ }^{30}$. Para reduzir o viés de memória, utilizou-se como período a presença do sintoma músculo-esquelético nos últimos três meses anteriores ao estudo, diferente de outros autores que usaram o período até um ano ${ }^{14}$. Um outro aspecto relevante foi um alto índice de Kappa entre o pré-teste e a sua replicação ${ }^{31}$, demonstrando uma excelente confiabilidade das respostas neste grupo de adolescentes de classe média brasileira. De fato, esta análise é uma medida de intensidade de concordância inter-observador ${ }^{31}$.

O presente estudo demonstrou uma alta prevalência de dor músculoesquelética referida igualmente em adolescentes obesos e eutróficos, principalmente dor em membros inferiores e dor no músculo trapézio. Uma freqüência de $40 \%$ de dor musculoesquelética em adolescentes eutróficos foi também observada em outro estudo nosso ${ }^{23}$. Nos Estados Unidos, $61 \%$ de 135 crianças e adolescentes obesos referiam pelo menos uma dor músculo-esquelética, não existindo neste estudo um grupo controle saudável ${ }^{10}$. Dor torácica foi mais frequentemente referida nos adolescentes eutróficos. Entretanto, o exame físico geral e específico não evidenciou alterações estruturais, incluindo síndrome miofascial.

A dor referida pelos adolescentes obesos e eutróficos deve ter sido preferencialmente aguda, transitória e localizada, e pode também ter sido superestimada por eles. O exame físico que confirmou as síndromes músculo-esqueléticas, e que habitualmente incluem doenças dolorosas crônicas, evidenciou estas alterações em apenas 18\% dos obesos que tinham dor. Dor crônica, ou seja, dor com duração acima de três meses foi apenas observada em um adolescente eutrófico com fibromialgia juvenil.

Outro aspecto interessante foi que alterações ortopédicas localizadas, principalmente encurtamento de quadríceps e geno valgo, foram evidenciadas em adolescentes obesos, assim como reportadas em outros

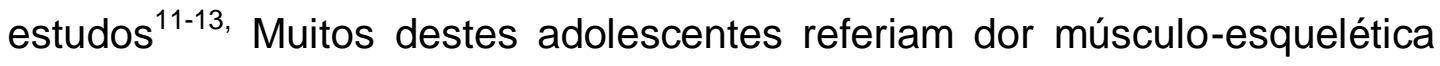


que deverão se intensificar com a manutenção da obesidade ao longo da vida, determinando alterações biomecânicas no sistema locomotor ${ }^{7}$. A persistência da obesidade nos adultos pode causar uma elevação da força de sustentação, ocasionando osteoartrite com erosões em joelhos e quadris $^{8,12}$, assim como um maior risco de fraturas ${ }^{11}$, dificuldades de movimento $^{32}$, epifisiólise ${ }^{11}$ e tíbia vara ${ }^{8}$. Estas alterações ortopédicas ao proporcionar maior adesão ao sedentarismo tenderiam a perpetuar o ganho de peso anormal destes pacientes ${ }^{6}$.

A exposição dos jovens aos aparelhos eletrônicos é um fenômeno crescente no mundo ${ }^{21}, 33-35 \mathrm{em}$ todas as classes econômicas ${ }^{36}$, como evidenciado no presente estudo. Interessantemente, computador e minigame foram utilizados menos frequentemente pelos obesos, e apenas 0 maior tempo de uso do videogame no domingo foi associado com dor nestes pacientes. Este aspecto também foi reportado em um estudo espanhol evidenciando que o excesso de peso foi explicado pela utilização de videogame durante o final de semana ${ }^{37}$.

O único fator associado à presença de dor músculo-esquelética em adolescentes obesos foi o sexo feminino, concordando com outros estudos $^{16,20,23}$. Na literatura médica o gênero feminino apresenta diferenças na percepção da dor, na forma de se relacionar com ela e, possivelmente, em seu maior relato. Além disto, o sexo feminino apresenta um limiar mais baixo, uma menor tolerância à dor ${ }^{23}$, e uma maior prevalência de dor em todas as faixas etárias ${ }^{16,20,23}$.

Este estudo tem limitações, pois não incluiu avaliação psicológica dos adolescentes. Distúrbios emocionais podem ser causa de dor ${ }^{20}$ e são fatores associados indiscutivelmente à obesidade na adolescência ${ }^{2,8}$. Um futuro estudo será realizado nesta mesma população, a partir de estudo radiográfico padronizado (avaliações do alinhamento das epífises, metáfises e diáfises dos membros inferiores), estadios puberais e das complicações metabólicas da obesidade.

Os pacientes obesos desse estudo apresentaram hipertensão arterial 
e elevados valores de IMC e de circunferência abdominal, conforme amplamente é reportado na literatura ${ }^{1,24}$. Entretanto, o peso excessivo não parece ter contribuído para a presença de queixas de dor músculoesquelética. É importante ressaltar que a população estudada esteve predominantemente no início da adolescência e que a obesidade crônica nesses jovens pacientes pode determinar alterações ósteo-articulares e de partes moles irreversíveis.

Um programa de atividade física supervisionada ou em grupo (como natação, corrida, caminhada, ciclismo etc) pode melhorar a dor, propiciar um fortalecimento muscular, melhorar a autoestima, e a qualidade de vida, conforme evidenciado em outras doenças crônicas pediátricas ${ }^{38,39}$. Orientações de ergonomia e relaxamento muscular devam ser ensinadas no ambiente escolar dos adolescentes e professores ${ }^{24,40}$.

Em conclusão, obesidade pode causar danos ao sistema ósteoarticular no início da adolescência, principalmente nos membros inferiores. Adolescentes obesas do sexo feminino relataram mais dor músculoesquelética, sugerindo que programas específicos para esse gênero devam ser desenvolvidos. Estudo longitudinal para avaliar o impacto da obesidade na biomecânica músculo-esquelética dos adolescentes será necessário.

\section{AGRADECIMENTOS}

Esse estudo teve apoio do Conselho Nacional de Desenvolvimento Científico e Tecnológico - CNPQ (Grant 300248/2008-3) e da Federico Foundation para CAAS. 


\section{REFERÊNCIAS}

1. Catenacci VA, Hill JO, Wyatt HR. The obesity epidemic. Clin Chest Med 2009;30:415-44.

2. Bruni V, Dei M, Peruzzi E, Seravalli V. The anorectic and obese adolescent. Best Pract Res Clin Obstet Gynaecol 2010;24:243-58.

3. Enes CC, Slater B. Obesity in adolescence and its main determinants. Rev Bras Epidemiol 2010;13:163-71.

4. In-Iw S, Biro FM. Adolescent Women and Obesity. J Pediatr Adolesc Gynecol 2011;24:58-61.

5. Wang Y, Monteiro C, Popkin BM. Trends of obesity and underweight in older children and adolescents in the United States, Brazil, China, and Russia. Am J Clin Nutr 2002;75:971-7.

6. Taylor ED, Theim KR, Mirch MC, Ghorbani S, Tanofsky-Kraff M, AdlerWailes DC, et al. Orthopedic complications of overweight in children and adolescents. Pediatrics 2006;117:2167-74.

7. Wearing SC, Hennig EM, Byrne NM, Steele JR, Hills AP. Musculoskeletal disorders associated with obesity: a biomechanical perspective. Obes Rev 2006;7:239-50.

8. Daniels SR. The consequences of childhood overweight and obesity. Future Child 2006;16:47-67.

9. de Sá Pinto AL, de Barros Holanda PM, Radu AS, Villares SM, Lima FR. Musculoskeletal findings in obese children. J Paediatr Child Health 2006;42:341-4.

10.Stovitz SD, Pardee PE, Vazquez G, Duval S, Schwimmer JB. Musculoskeletal pain in obese children and adolescents. Acta Paediatr 2008;97:489-93.

11.Chan G, Chen CT. Musculoskeletal effects of obesity. Curr Opin Pediatr. 2009;21:65-70.

12. Ells LJ, Lang R, Shield JP, Wilkinson JR, Lidstone JS, Coulton S, et al. Obesity and disability - a short review. Obes Rev 2006;7:341-5.

13. Gettys FK, Jackson JB, Frick SL. Obesity in pediatric orthopaedics. 
Orthop Clin North Am 2011;42:95-105.

14. Fassa AG, Facchini LA, Dall'Agnol MM, Christiani DC. Child labor and musculoskeletal disorders: the Pelotas (Brazil) epidemiological survey. Public Health Rep 2005;120:665-73.

15. Boström M, Dellve L, Thomée S, Hagberg M. Risk factors for generally reduced productivity--a prospective cohort study of young adults with neck or upper-extremity musculoskeletal symptoms. Scand J Work Environ Health 2008;34:120-32.

16.Grimby-Ekman A, Andersson EM, Hagberg M. Analyzing musculoskeletal neck pain, measured as present pain and periods of pain, with three different regression models: a cohort study. BMC Musculoskelet Disord 2009;19;10:73.

17.Smith L, Louw Q, Crous L, Grimmer-Somers K. Prevalence of neck pain and headaches: impact of computer use and other associative factors. Cephalalgia 2009;29:250-7.

18. Jacobs K, Hudak S, McGiffert J. Computer-related posture and musculoskeletal discomfort in middle school students. Work 2009;32:275-83.

19. Kelly G, Dockrell S, Galvin R. Computer use in school: its effect on posture and discomfort in schoolchildren. Work 2009;32:321-8.

20.Diepenmaat AC, van der Wal MF, de Vet HC, Hirasing RA. Neck/shoulder, low back, and arm pain in relation to computer use, physical activity, stress, and depression among Dutch adolescents. Pediatrics 2006;117:412-6.

21.Zapata AL, Moares AJ, Leone C, Doria-Filho U, Silva CA. Pain and musculoskeletal pain syndromes related to computer and video game use in adolescents. Eur J Pediatr 2006;165:408-14.

22. Must A, Dallal GE, Dietz WH. Reference data for obesity: 85th and 95th percentiles of body mass index (wt/ht2) and triceps skinfold thickness. Am J Clin Nutr 1991; 53:839-46.

23.Zapata AL, Moraes AJP, Leone C, Doria-Filho U, Silva CA. Pain and musculoskeletal pain syndromes in adolescents. J Adolesc Health 
2006; 38:769-71.

24. Silva CA, Zapatta AL, Moares AJ, Doria-Filho U, Leone C. Utilização do computador e de jogos eletrônicos e avaliação da ergonomia com uso do computador em adolescentes de uma escola privada na cidade de São Paulo. Rev Paul Pediatria 2006;24:104-10.

25. Almeida PM, Wickerrhauser $\mathrm{H}$. Critério de classe econômica da Associação Brasileira de Anunciantes (ABA) e Associação Brasileira dos Institutos de Pesquisa de Mercado (ABIPEME) 1991, p 1-29.

26. National High Blood Pressure Education Program Working Group on High Blood Pressure in Children and Adolescents. The fourth report on the diagnosis, evaluation, and treatment of high blood pressure in children and adolescents. Pediatrics 2004;114:555-76.

27. Hoppenfeld S, Hutton R. Physical examination of the spine \& extremities. In: Hoppenfeld S, Hutton R, eds. Physical Examination of the Knee, Ankle,Foot and Lumbar Spine. New York: Prentice Hall, 1976;171-264.

28. Wolfe F, Smythe HA, Yunus MB, Bennett RM, Bombardier C, Goldenberg DL, et al. The American College of Rheumatology 1990 criteria for the classification of fibromyalgia. Report of multicentre criteria committee Arthritis Rheum 1990;33:160-72.

29. Beighton P, Solomon L, Soskolne CL. Articular mobility in an African population. Ann Rheum Dis 1973;32:413-8.

30. Mikkelsson M, Salminen JJ, Kautiainen H. Non-specific musculoskeletal pain in preadolescents: prevalence and 1-year persistence. Pain 1997;73:29-35.

31.Landis JR, Koch GG. The measurement of observer agreement for categorical data. Biometrics 1977;33:159-74.

32. Tsuritani I, Honda R, Noborisaka Y, Ishida M, Ishizaki M, Yamada Y. Impact of obesity on musculoskeletal pain and difficulty of daily movements in Japanese middle-aged women. Maturitas 2002;42:2330.

33. Van der Voort T, Beentjes WWJ, Bovill Ml. Y. Young people's 
ownership and uses of new and old forms of media in Britain and The Netherlands. Eur J Commun 1998;13:457-77.

34.Johnsson-Smaragdi U, d'Haenens L, Krotz F. Patterns of old and new media use among young people in Flanders,Germany and Sweden. Eur J Commun 1998;13:479-501.

35. Roberts DF. Media and youth: access, exposure and privatization. J Adolesc Health 2000;27:8-14.

36. Devís-Devís J, Peiró-Velert C, Beltrán-Carrillo VJ, Tomás JM. Screen media time usage of 12-16 year-old Spanish school adolescents: Effects of personal and socioeconomic factors, season and type of day. J Adolesc 2009;32:213-31.

37. Vicente-Rodríguez G, Rey-López JP, Martín-Matillas M, Moreno LA, Wärnberg J, Redondo C, et al. Television watching, videogames, and excess of body fat in Spanish adolescents: the AVENA study. Nutrition 2008;24:654-62.

38. Gualano B, Sá Pinto AL, Perondi B, Leite Prado DM, Omori C, Almeida RT, et al. Evidence for prescribing exercise as treatment in pediatric rheumatic diseases. Autoimmun Rev 2010;9:569-73.

39. Omori C, Prado DM, Gualano B, Sallum AM, Sá-Pinto AL, Roschel H, et al. .Responsiveness to exercise training in juvenile dermatomyositis: a twin case study. BMC Musculoskelet Disord 2010;11:270.

40. Campos LM, Silva CA. Como minimizar a má influência do computador. Rev Paulista Reumatol 2008;7:5-7. 
Tabela 1 - Dados demográficos, dados antropométricos, pressão arterial (PA), prática esportiva, síndromes músculo-esqueléticas e alterações ortopédicas em adolescentes obesos versus eutróficos.

\begin{tabular}{|c|c|c|c|}
\hline Variáveis & $\begin{array}{l}\text { Obesos } \\
(n=100)\end{array}$ & $\begin{array}{l}\text { Eutróficos } \\
(n=100)\end{array}$ & $\mathbf{P}$ \\
\hline \multicolumn{4}{|l|}{ Dados demográficos } \\
\hline Idade atual, anos & $12,6(10-18,2)$ & $13(10-19)$ & 0,051 \\
\hline Gênero feminino & $48(52)$ & $60(60)$ & 0,089 \\
\hline Classe socioeconômica, C e & $71(71)$ & $62(62)$ & 0,231 \\
\hline \multirow{2}{*}{\multicolumn{4}{|c|}{$\begin{array}{l}\text { D } \\
\text { Dados antropométricos }\end{array}$}} \\
\hline & & & \\
\hline $\mathrm{IMC}, \mathrm{kg} / \mathrm{m}^{2}$ & $\begin{array}{l}29,45(23,7- \\
45,4)\end{array}$ & $18,8(14,8-24,1)$ & 0,0001 \\
\hline $\begin{array}{l}\text { Circunferência abdominal, cm } \\
\text { PA }\end{array}$ & $95(69,5-123)$ & $69(55-83)$ & 0,0001 \\
\hline Hipertensão arterial sistêmica & $19(21)$ & $3(3)$ & 0,0004 \\
\hline PA sistólica, mmHg & $110(80-140)$ & $100(70-130)$ & 0,0001 \\
\hline PA diastólica, $\mathrm{mmHg}$ & $70(50-90)$ & $60(40-80)$ & 0,0001 \\
\hline \multicolumn{4}{|l|}{ Prática esportiva } \\
\hline Educação física $\geq 2$ vezes/sen & $92(92)$ & $92(92)$ & 1,0 \\
\hline Esportes $\geq 2$ vezes/semana & $26(26)$ & $27(27)$ & 1,0 \\
\hline Dor músculo-esquelética & $44(44)$ & $56(56)$ & 0,09 \\
\hline Dor cervical & $6(6)$ & $10(10)$ & 0,435 \\
\hline Dor em membros superiores & $3(3)$ & $10(10)$ & 0,082 \\
\hline Dor torácica & $2(2)$ & $13(13)$ & 0,005 \\
\hline Dor em músculo trapézio & $16(16)$ & $17(17)$ & 1,0 \\
\hline Dor lombar & $0(0)$ & $4(4)$ & 0,121 \\
\hline Dor em membros inferiores & $23(23)$ & $22(22)$ & 1,0 \\
\hline $\begin{array}{l}\text { Síndromes músculo- } \\
\text { esqueléticas }\end{array}$ & $12(12)$ & $16(16)$ & 0,541 \\
\hline Fibromialgia juvenil & $0(0)$ & $1(1)$ & 1,0 \\
\hline SHAB & $0(0)$ & $2(2)$ & 0,497 \\
\hline Síndrome miofascial & $0(0)$ & $1(1)$ & 1,0 \\
\hline Tendinite & $9(9)$ & $3(3)$ & 0,134 \\
\hline Epicondilite & $3(3)$ & $11(11)$ & 0,027 \\
\hline Bursite & $0(0)$ & $0(0)$ & 1,0 \\
\hline Alterações ortopédicas & $98(98)$ & $76(76)$ & 0,0001 \\
\hline Escoliose & $30(30)$ & 33 (33) & 0,761 \\
\hline Encurtamento de quadríceps & $89(89)$ & $44(44)$ & 0,0001 \\
\hline Geno valgo & $87(87)$ & $24(24)$ & 0,0001 \\
\hline Geno varo & $1(1)$ & $8(8)$ & 0,0349 \\
\hline Geno recurvatum & $1(1)$ & $0(0)$ & 1,0 \\
\hline Encurtamento de isquiotibiais & $6(6)$ & $1(1)$ & 0,054 \\
\hline Hálux valgo & $1(1)$ & $0(0)$ & 1,0 \\
\hline
\end{tabular}




\begin{tabular}{|c|c|c|c|}
\hline Variáveis & $\begin{array}{l}\text { Obesos } \\
(n=100)\end{array}$ & $\begin{array}{c}\text { Eutróficos } \\
(\mathrm{n}=100)\end{array}$ & $\mathbf{P}$ \\
\hline \multicolumn{4}{|l|}{ Computador } \\
\hline Uso do computador & $69(69)$ & $79(79)$ & 0,107 \\
\hline Disponibilidade domiciliar & $54(54)$ & $68(68)$ & 0,042 \\
\hline Uso de computador próprio & $24(24)$ & $36(36)$ & 0,064 \\
\hline Uso de Laptop próprio & $8(8)$ & $14(14)$ & 0,233 \\
\hline Uso no dia anterior & $32(32)$ & $48(48)$ & 0,015 \\
\hline Idade de início do uso, anos & $10(5-15)$ & $11(5-16)$ & 0,01 \\
\hline Tempo de uso no dia anterior, min & $30(0-360)$ & $60(0-720)$ & 0,0001 \\
\hline Tempo de uso nos sábados, min & $1(0-720)$ & $60(0-600)$ & 0,001 \\
\hline Tempo de uso nos domingos, min & $0(0-720)$ & $30(0-720)$ & 0,02 \\
\hline Tempo de uso na semana, dias & $2(0-7)$ & $3(0-7)$ & 0,077 \\
\hline \multicolumn{4}{|l|}{ VG } \\
\hline Uso do VG & $39(39)$ & $43(43)$ & 0,605 \\
\hline Disponibilidade domiciliar & $32(32)$ & $35(35)$ & 0,617 \\
\hline Uso de VG próprio & $21(21)$ & $24(24)$ & 0,638 \\
\hline Uso do VG no dia anterior & $12(12)$ & $12(12)$ & 1,0 \\
\hline Tempo de uso no dia anterior, min & $0(0-720)$ & $0(0-360)$ & 0,459 \\
\hline Tempo de uso no sábado, min & $0(0-720)$ & $0(0-720)$ & 0,933 \\
\hline Tempo de uso no domingo, min & $0(0-720)$ & $0(0-720)$ & 0,833 \\
\hline Tempo de uso na semana, dias & $0(0-7)$ & $0(0-7)$ & 0,65 \\
\hline \multicolumn{4}{|l|}{ MG } \\
\hline Uso do MG & $2(2)$ & $11(11)$ & 0,003 \\
\hline Uso do MG no dia anterior & $2(2)$ & $3(3)$ & 1,0 \\
\hline Tempo de uso na semana, dias & $0(0-5)$ & $0(0-7)$ & 0,035 \\
\hline Tempo de uso no dia anterior, min & $0(0-15)$ & $0(0-720)$ & 0,637 \\
\hline
\end{tabular}

Os resultados são apresentados em $n$ (\%) e mediana (variação), min - minutos. 
Tabela 3 - Dados demográficos, dados antropométricos, pressão arterial (PA), prática esportiva, síndromes músculo-esqueléticas e alterações ortopédicas em adolescentes obesos com dor versus obesos sem dor.

\begin{tabular}{|c|c|c|c|}
\hline Variáveis & $\begin{array}{c}\text { Obesos com } \\
\text { dor } \\
(n=44)\end{array}$ & $\begin{array}{c}\text { Obesos sem } \\
\text { dor } \\
(n=56)\end{array}$ & $\mathbf{P}$ \\
\hline \multicolumn{4}{|l|}{ Dados demográficos } \\
\hline Idade atual, meses & $12,5(10-19)$ & $\begin{array}{l}12,7(10,2- \\
17,2)\end{array}$ & 0,692 \\
\hline Gênero feminino & $26(59)$ & $22(39)$ & 0,048 \\
\hline $\begin{array}{l}\text { Classe socioeconômica, C } \\
\text { ou D }\end{array}$ & $32(73)$ & $39(70)$ & 0.829 \\
\hline \multicolumn{4}{|l|}{ Dados antropométricos } \\
\hline IMC, $\mathrm{kg} / \mathrm{m}^{2}$ & $\begin{array}{c}29,2(23,7- \\
43,5)\end{array}$ & $\begin{array}{l}29,5(24,1- \\
45,4)\end{array}$ & 0,981 \\
\hline $\begin{array}{l}\text { Circunferência abdominal, cm } \\
\text { PA }\end{array}$ & $94(69,5-117)$ & $96,25(82-123)$ & 0,352 \\
\hline $\begin{array}{l}\text { Hipertensão arterial } \\
\text { sistêmica }\end{array}$ & $9(21)$ & $10(18)$ & 0,8 \\
\hline PA sistólica, mmHg & $110(80-140)$ & $110(80-130)$ & 0,432 \\
\hline PA diastólica, mmHg & $70(50-90)$ & $70(50-90)$ & 0,945 \\
\hline \multicolumn{4}{|l|}{ Prática esportiva } \\
\hline Educação física $\geq 2$ & $41(93)$ & $51(91)$ & 1,0 \\
\hline \multicolumn{4}{|l|}{ vezes/semana } \\
\hline Esportes $\geq 2$ vezes/semana & $11(25)$ & $15(27)$ & 1,0 \\
\hline $\begin{array}{l}\text { Síndromes músculo- } \\
\text { esqueléticas }\end{array}$ & $8(18)$ & $4(7)$ & 0,124 \\
\hline Fibromialgia juvenil & $0(0)$ & $0(0)$ & 1,0 \\
\hline SHAB & $0(0)$ & $0(0)$ & 1,0 \\
\hline Síndrome miofascial & $0(0)$ & $0(0)$ & 1,0 \\
\hline Tendinite & $6(14)$ & $3(5)$ & 0,176 \\
\hline Epicondilite & $2(4)$ & $1(2)$ & 0,581 \\
\hline Alterações ortopédicas & $44(100)$ & $54(96)$ & 0,502 \\
\hline Escoliose & $11(25)$ & $19(34)$ & 0,384 \\
\hline Encurtamento de quadríceps & $39(89)$ & $50(89)$ & 1,0 \\
\hline Geno valgo & $41(93)$ & $46(82)$ & 0,1380 \\
\hline Geno varo & $1(2)$ & $0(0)$ & 0,44 \\
\hline Geno recurvatum & $1(2)$ & $0(0)$ & 0,44 \\
\hline Encurtamento de & $1(2)$ & $5(9)$ & 0,225 \\
\hline \multicolumn{4}{|l|}{ ísquiotibiais } \\
\hline Hálux valgo & $1(2)$ & $0(0)$ & 0,44 \\
\hline
\end{tabular}




\begin{tabular}{|c|c|c|c|}
\hline Variáveis & $\begin{array}{c}\text { Obesos } \\
\text { com dor } \\
(n=44)\end{array}$ & $\begin{array}{c}\text { Obesos sem } \\
\text { dor } \\
(n=56)\end{array}$ & $\mathbf{P}$ \\
\hline \multicolumn{4}{|l|}{ Computador } \\
\hline Uso do computador & $30(68)$ & $39(70)$ & 1,0 \\
\hline Disponibilidade domiciliar & $24(54)$ & $30(54)$ & 1,0 \\
\hline Uso de computador próprio & $12(27)$ & $12(21)$ & 0,638 \\
\hline Uso de Laptop próprio & $6(14)$ & $2(4)$ & 0,133 \\
\hline Uso no dia anterior & $12(27)$ & $20(36)$ & 0,396 \\
\hline Idade de início do uso, anos & $10(5-10,8)$ & $10(3-15)$ & 0,734 \\
\hline Tempo de uso no dia anterior, & $20(0-360)$ & $30(0-300)$ & 0,558 \\
\hline Tempo de uso nos sábados, $n$ & $1(0-720)$ & $45(0-720)$ & 0,14 \\
\hline Tempo de uso nos domingos, & $1(0-720)$ & $30(0-720)$ & 0,646 \\
\hline \multicolumn{4}{|l|}{ VG } \\
\hline Uso do VG & $17(39)$ & $22(39)$ & 1,0 \\
\hline Disponibilidade domiciliar & $16(36)$ & $16(29)$ & 0,518 \\
\hline Uso de VG próprio & $9(21)$ & $12(21)$ & 1,0 \\
\hline Uso do VG no dia anterior & $5(11)$ & $7(13)$ & 1,0 \\
\hline Tempo de uso no dia anterior, & $0(0-720)$ & $0(0-120)$ & 0,123 \\
\hline Tempo de uso nos sábados, $n$ & $0(0-720)$ & $0(0-180)$ & 0,128 \\
\hline Tempo de uso nos domingos, & $0(0-720)$ & $0(0-240)$ & 0,028 \\
\hline Tempo de uso na semana, dia & $0(0-7)$ & $(0-7)$ & 0,655 \\
\hline \multicolumn{4}{|l|}{ MG } \\
\hline Uso do MG & $1(2)$ & $1(2)$ & 1,0 \\
\hline Uso do MG no dia anterior & $1(2)$ & $1(2)$ & 1,0 \\
\hline Tempo de uso na semana, dia & $0(0-5)$ & $0(0-2)$ & 0,775 \\
\hline Tempo de uso no dia anterior, & $0(0-15)$ & $0(0-2)$ & 0,713 \\
\hline
\end{tabular}

Os resultados são apresentados em $\mathrm{n}$ (\%) e mediana (variação), min - minutos. 